\title{
The equivalence principle and the Moon
}

\author{
Thibault Damour \\ Institut des Hautes Etudes Scientifiques, 91440 Bures sur Yvette, France \\ and Département d'Astrophysique Relativiste et de Cosmologie, Observatoire de Paris, \\ Centre National de la Recherche Scientifique, 92195 Meudon CEDEX, France \\ David Vokrouhlický⿴囗十 \\ Observatoire de la Côte d'Azur, Département CERGA, Avenue N. Copernic, \\ 06130 Grasse, France
}

(September 3, 2018)

\begin{abstract}
The perturbation of the lunar motion caused by a hypothetical violation of the equivalence principle is analytically worked out in terms of power series à la Hill-Brown. The interaction with the quadrupolar tide is found to amplify the leading order term in the synodic range oscillation by more than $62 \%$. Confirming a recent suggestion of Nordtvedt, we find that this amplification has a pole singularity for an orbit beyond the lunar orbit. This singularity is shown to correspond to the critical prograde orbit beyond which, as found by Hénon, Hill's periodic orbit becomes exponentially unstable. It is suggested that ranging between prograde and retrograde orbits around outer planets might provide future high precision orbital tests of the equivalence principle. It is argued that, within the context of string-derived non-Einsteinian theories, the theoretical significance of orbital tests of the universality of free fall is to measure the basic coupling strength of some scalar field through compositiondependent effects. Present Lunar Laser Ranging data yield the value $\bar{\gamma}=$ $(-1.2 \pm 1.7) \times 10^{-7}$ for the effective Eddington parameter $\bar{\gamma} \equiv \gamma-1$ measuring this coupling strength.
\end{abstract}




\section{INTRODUCTION}

Gravity seems to enjoy a remarkable universality property: all bodies are experimentally found to fall with the same acceleration in an external gravitational field, independently of their mass and composition. Although Galileo [1] was the first [2] to suggest in a clear and general way that this property of universality of free fall might hold true, it was left to Newton [3] to realize the remarkable conceptual status of this universality: exact proportionality between a particular force (the weight) and the general dynamical measure of inertia (the mass). Newton went further in performing the first precise laboratory tests of the universality of free fall (pendulum experiments; precision $\sim 10^{-3}$ ). It is less well known that Newton went even further and suggested to test the universality of free fall of celestial bodies by looking for a possible miscentering of the orbits of satellites around Jupiter, Saturn and the Earth [4. More precisely, Newton considers a possible violation of the ratio weight $(w)$ over mass $(m)$, i.e.

$$
\bar{\delta}_{12} \equiv \frac{(w / m)_{1}}{(w / m)_{2}}-1 \neq 0,
$$

where 1 labels a satellite and 2 a planet, the weights $w_{1}, w_{2}$ being the gravitational forces exerted by the Sun (label 3). He says, without giving any details, that he has found "by some computations" that the center $\mathbf{x}_{c}$ of the orbit of the satellite 1 around the planet 2 will be displaced (in the Sun-planet direction and away from the Sun if $\bar{\delta}_{12}>0$ ) by the amount

$$
\delta\left|\mathbf{x}_{c}-\mathbf{x}_{3}\right|=+\frac{1}{2} \bar{\delta}_{12} a^{\prime}
$$

where $a^{\prime}$ denotes the radius of the orbit of the planet 2 around the Sun. In modern phraseology, one can say that Newton predicted a "polarization" of the satellite's orbit in the Sun-planet direction (away from the Sun if $\bar{\delta}_{12}>0$ ). Then Newton used his theoretical estimate (1.2) to conclude from the observed good centering of the orbits of the satellites of Jupiter that $\left|\bar{\delta}_{12}\right|<10^{-3}$, a number comparable to the result of his pendulum experiments. Actually, this upper limit obtained from Jovian satellites is wrong, as Newton's theoretical estimate (1.2) is incorrect both in magnitude (being a gross overestimate in general) and in sign (see below). We could not find any information about Newton's original calculations in his published papers. It is surprising that Newton did not remark that, as a consequence of his estimate (1.2), a value $\left|\bar{\delta}_{12}\right|=10^{-3}$ would also entail an unacceptably large polarization (one fifth) of the Moon's orbit.

As far as we are aware, Laplace was the first to realize that the best celestial system to test a possible violation of the universality of free fall (1.1) is the Earth-Moon system $(1=$ Moon, $2=$ Earth $)$. In [5] he derived a rough estimate of the main observable effect of

$\bar{\delta}_{12}$ on the angular motion of the Moon. Then, he noticed that even a very small $\bar{\delta}_{12} \neq 0$ would spoil the agreement linking his theoretical derivation of the "parallactic inequality" of the Moon, the set of observations of the lunar motion, and the direct measurements of the solar parallax. He concluded that an upper bound to the fractional difference in acceleration of the Moon and the Earth toward the Sun is

$$
\left|\bar{\delta}_{12}\right|<\frac{1}{3410000} \simeq 2.9 \times 10^{-7} .
$$


It is remarkable that the limit (1.3) is much better than the limit $\left(\left|\bar{\delta}_{A B}\right|<2 \times 10^{-5}\right)$ obtained some years later by Bessel through improved pendulum experiments [6], and has been superseded (though not by much) only by the work of Eötvös in $1890\left(\left|\bar{\delta}_{A B}\right|<5 \times 10^{-8}\right.$; [7]). [The later results of Eötvös, Pekár and Fekete improved the bound to $3 \times 10^{-9}$; [8].] As we discuss in Appendix D, in spite of some obscurities in his reasonings and the lack of a fully accurate calculation of the effect of $\bar{\delta}_{12}$ in longitude, Laplace's final bound (1.3) turns out to be a conservative upper limit, given the information he had.

In 1907, Einstein [9] deepened the conceptual implications of the property of universality of free fall by raising it to the level of a "hypothesis of complete physical equivalence" between a gravitational field and an accelerated system of reference. This heuristic hypothesis was used very successfully by Einstein in his construction of the theory of general relativity, and became later enshrined in the name "principle of equivalence".

Within the context of relativistic gravitational theories, the use of the Moon as a sensitive probe of a possible violation of the equivalence principle for massive, self-gravitating bodies has been rediscovered by Nordtvedt in 1968 [10]. His idea was that self-gravitational energies might couple non universally to an external gravitational field in theories having a different structure than general relativity [11], [12]. [Let us note that Dicke had mentioned this possibility earlier [13], [14], [15] but had not explored its consequences in detail.] Anyway, Nordtvedt, unaware both of the old ideas of Newton and Laplace, and of the more recent ones of Dicke, realized that the planned Lunar Laser Ranging (LLR) experiment was providing an exquisitely sensitive tool for testing the universality of free fall of massive bodies [10]. Performing a first-order perturbation analysis of the lunar orbit (assumed circular and planar) in presence of a violation of the equivalence principle, $\bar{\delta}_{12} \neq 0$ (see Eq. (1.1) with the labels 1 and 2 denoting the Moon and the Earth, respectively), he provided the first analytical estimate of the corresponding range oscillation:

$$
(\delta r)^{(1)}=C^{(1)} \bar{\delta}_{12} a^{\prime} \cos \left[\left(n-n^{\prime}\right) t+\tau_{0}\right],
$$

with

$$
C^{(1)}=\frac{1+2 n /\left(n-n^{\prime}\right)}{n^{2}-\left(n-n^{\prime}\right)^{2}} n^{\prime 2} .
$$

Here, $n$ denotes the (mean) sidereal angular velocity of the Moon around the Earth, $n^{\prime}$ the (mean) sidereal angular velocity of the Earth around the Sun, and $a^{\prime}$ denotes the radius of the orbit of the Earth around the Sun (assumed circular). The angle $\tau \equiv\left(n-n^{\prime}\right) t+\tau_{0}$ is equal to the difference between the mean longitude of the Moon and the mean longitude of the Sun (as seen from the Earth). For completeness we derive Eqs. (1.4), (1.5) in Appendix A.

Let us note in passing that the $\cos \tau$ dependence of the range oscillation (1.4) is equivalent (when disregarding the perturbations of the motion in longitude) to displacing the center $\mathbf{x}_{c}$ of a circular lunar orbit in the Earth-Sun direction (toward the Sun if $\bar{\delta}_{12}>0$ ) by the amount

$$
\delta\left|\mathbf{x}_{c}-\mathbf{x}_{3}\right|=-C^{(1)} \bar{\delta}_{12} a^{\prime}
$$

The result (1.2) of Newton can therefore be viewed as implying a range oscillation of the type (1.4) with $C^{\text {Newton }}=-\frac{1}{2}$, independently of $n$ and $n^{\prime}$. By contrast, the first order 
estimate (1.5) contains the small dimensionless parameter

$$
m=\frac{n^{\prime}}{n-n^{\prime}},
$$

which is $m \simeq 1 / 12.3687$ for the Moon and much smaller for the (Galilean) satellites of Jupiter (e.g. $m \simeq 3.86 \times 10^{-3}$ for Jupiter IV). More precisely, Eq. (1.5) can be rewritten as

$$
C^{(1)}=\frac{3}{2} m \frac{1+\frac{2}{3} m}{1+\frac{1}{2} m}=\frac{3}{2} m\left[1+\frac{1}{6} m-\frac{1}{12} m^{2}+\cdots\right] .
$$

In 1973, Nordtvedt [16] suggested that a more accurate value of the coefficient $C$ in the $\cos \tau$ (or "synodic") range oscillation (1.4) would be obtained by replacing, in the denominator of $C^{(1)}$, Eq. (1.5), the angular velocity $n$ by the frequency of radial perturbations: $n_{\text {rad }}=\dot{l}=\overline{\mathrm{c}} n$ where the perturbation series giving $\overline{\mathrm{c}}$ reads (see e.g. [17], [18])

$$
\overline{\mathrm{c}}=1-\frac{\dot{\varpi}}{n}=1-\frac{3}{4} m^{2}-\frac{177}{32} m^{3}-\cdots .
$$

[In the case of the Moon, the series (1.9) is very slowly convergent. The full value of $1-\overline{\mathrm{c}} \simeq 0.008572573$ [17] is more than twice the lowest-order correction $\frac{3}{4} m^{2}$.] The correction of Ref. [16] amounts numerically to increasing the first-order result (1.5) by about $13 \%$.

In 1981, Will [19] tried, more systematically, to estimate the higher-order corrections in the coefficient $C$ due to the mixing between the perturbation (1.4) at frequency $n-n^{\prime}$ and the tidal perturbations at frequencies 0 and $2\left(n-n^{\prime}\right)$. He suggested that the first order result should be multiplied by a factor $1+2 n^{\prime} / n=1+2 m+\mathcal{O}\left(m^{2}\right)$, i.e. amplified by about $15 \%$. As a result of these (coincidentally equivalent) prescriptions, the literature on the "Nordtvedt effect" [20], [21], [19], [22], [23] has, for many years, used as standard estimate for the range oscillation $\delta r=C \bar{\delta}_{12} a^{\prime} \cos \tau$ a value $C \simeq 1.14 C^{(1)}$ [corresponding to about $9.3 \eta \cos \tau$ meters in metrically-coupled theories; see below].

Actually, both modifications (suggested in [16] and [19]) of the first-order result fall short of giving an accurate estimate of the effects due to higher powers of $m$. In fact, they do not even give correctly the second order in $m$. For completeness, we compute in Appendix A, by the standard perturbation theory of de Pontécoulant [24], the contribution at order $\mathcal{O}\left(\mathrm{m}^{2}\right)$ and find that it amounts to multiplying the first-order result by $1+\frac{9}{2} m+\mathcal{O}\left(m^{2}\right)$, i.e.

$$
\begin{aligned}
C^{(1)}+C^{(2)} & =\left[1+\frac{9}{2} m+\mathcal{O}\left(m^{2}\right)\right] C^{(1)} \\
& =\frac{3}{2} m\left[1+\frac{14}{3} m+\mathcal{O}\left(m^{2}\right)\right] .
\end{aligned}
$$

By contrast, 16] and [19] would give $\frac{11}{12}$ and $\frac{13}{6}$, respectively, for the coefficient of $m$ in the correcting factor within square brackets in the second Eq. (1.10). Note that $\frac{14}{3} m=37.7$

1 This is the version of the small parameter which is appropriate to our Hill-Brown treatment. Beware of the fact that the more traditional perturbation approaches denote by the letter $m$ the quantity $\bar{m} \equiv n^{\prime} / n$. 
$\%$ for the Moon. Recently, Nordtvedt [25] argued that the full perturbation series in powers of $m$ (created by the interaction with the orbit's tidal deformation) will cause a rather large numerical amplification of the synodic oscillation (1.4). However, he gave only an incomplete theoretical analysis of this amplification. The only literal result he gives (his Eq. (2.33)) is equivalent to the second-order effect derived in Appendix A and leading to Eq. (1.10). We could not extract from his paper a precise value for the full effect of the $m$-series.

The aim of the present paper is to provide, for the first time, a full-fledged Hill-Brown analytical treatment of the orbital perturbations caused by a violation of the equivalence principle. Our results will allow us notably to give a precise numerical value for the full range oscillation in the case of the actual Moon?. Namely, we obtain below

$$
\delta r=2.9427 \times 10^{12} \bar{\delta}_{12} \cos \tau \mathrm{cm},
$$

corresponding to a full coefficient $C=\frac{3}{2} m \times 1.62201$ which is larger than the first-order value (1.8) by more than $60 \%$. More generally, we shall be able to discuss in detail the dependence on $m$ of the range oscillation: see Eqs. (2.60)-(2.62) and Appendix B. These results are summarized in Fig.1 below. Our results confirm the suggestion of Ref. [25] that when $m$ increases (corresponding to prograde orbits beyond the actual lunar orbit) the $\cos \tau$ range oscillation eventually becomes resonant and is (formally) infinitely amplified. We have some doubts, however, about the practical utility of such a resonant orbit, notably because we show that it occurs precisely at the value

$$
m=m_{c r}=0.1951039966 \ldots
$$

(corresponding to a sidereal period $T_{c r}=m_{c r} /\left(1+m_{c r}\right)$ yr $=1.95903$ month) where the orbit becomes exponentially unstable. Armed with our theoretical understanding of the $m$-dependence of the $\cos \tau$ oscillation, we suggest other orbits that might be practically interesting (retrograde orbits, orbits around outer planets). Finally, we emphasize that within the context of modern unified theories the most probable theoretical significance of orbital tests of the universality of free fall is the same as that of laboratory tests, namely to measure, through composition-dependent effects, the strength of the coupling to matter of some long range scalar field(s). The basic measure of this coupling strength is embodied in an effective Eddington parameter $\bar{\gamma} \equiv \gamma-1$ which governs both the standard post-Newtonian effects (including the violation of the strong equivalence principle $\propto \eta \equiv 4 \bar{\beta}-\bar{\gamma}$ ) and the composition-dependent couplings (violation of the weak equivalence principle). Actually, string theory suggests that the former contribution (proportional to the gravitational binding energy) is negligible compared to the one due to a violation of the weak equivalence principle. Interpreting the latest LLR observational results within a recently studied class of stringderived theoretical models, we conclude that present orbital tests give the excellent constraint $\bar{\gamma}=(-1.2 \pm 1.7) \times 10^{-7}$. [This limit is comparable to the (similarly interpreted) constraint coming from laboratory tests of the weak equivalence principle: $\bar{\gamma}=(-0.8 \pm 1.0) \times 10^{-7}$.]

\footnotetext{
${ }^{2}$ Note, however, that we consider only the Main Lunar Problem, i.e. that we neglect the terms proportional to the squares of the lunar and solar eccentricities, and to the square of the lunar inclination, which are expected to modify our numerical estimates by $\lesssim 1 \%$.
} 
The plan of this paper is as follows. Section II presents our Hill-Brown approach and gives the analytical results obtained with it. Section III discusses the physical consequences of our results. Many technical details are relegated to some Appendices: Appendix A presents the standard de Pontécoulant treatment of lunar theory and uses it to derive the second-order result (1.10), Appendix B gives some details of our Hill-Brown treatment, Appendix C treats the link between certain commensurabilities of frequencies, linear instability and the presence of pole singularities in perturbed motions, and finally Appendix D discusses Laplace's derivation of the remarkably good limit (1.3) on $\bar{\delta}_{12}$.

\section{HILL-BROWN TREATMENT OF EQUIVALENCE-PRINCIPLE-VIOLATION EFFECTS}

\section{A. Introduction}

Relativistic effects in the lunar motion have been investigated by many authors. The pioneers in this field are de Sitter [26] (who computed the general relativistic contributions to the secular motions of the lunar perigee and node as observed in a global, barycentric frame) and Brumberg [27] (who gave a comprehensive Hill-Brown treatment of the post-Newtonian 3-body problem). Later works studied non-Einsteinian effects, notably those associated with the Eddington post-Newtonian parameters $\beta$ and $\gamma$. The most comprehensive and accurate analytical study of post-Newtonian effects in the lunar motion (described in a barycentric frame) is due to Brumberg and Ivanova [18. For general accounts and more references see the books [28] and [29]. Let us also mention the semi-analytical treatment of the general relativistic perturbations of the Moon by Lestrade and Chapront-Touzé [30].

However, apart from the work of Nordtvedt [10], [16], [25], the studies of non-Einsteinian effects in the lunar motion have not considered the effect of a violation of the equivalence principle. The results of the present paper can therefore be considered as a completion of Ref. [18] which gave an accurate Hill-Brown theory of all the other Einsteinian and nonEinsteinian effects. In fact, as pointed out long ago by Nordtvedt, the effects of a violation of the universality of free fall are the most prominent non-Einsteinian effects in the lunar orbit, and therefore deserve an accurate study. Indeed, most of the non-Einsteinian effects are non-null effects, i.e. correspond to modifications proportional to $\bar{\beta} \equiv \beta-1$ or $\bar{\gamma} \equiv \gamma-1$ of observable relativistic effects (as seen in a local, geocentric frame) predicted by Einstein's theory. As the latter are at the few centimeter level [31], [28], [32], [25], which is the precision of the LLR data, they can be of no use for measuring $\bar{\beta}$ and $\bar{\gamma}$ at an interesting level (say $<10^{-2}$ ). An exception must be made for secular effects and for the parameters describing the temporal and spatial transformation linking a local, geocentric frame to a global, barycentric one, e.g. the parameters entering the de Sitter-Fokker ("geodetic") precession. [Recent work [33 concludes that geodetic precession alone constraints $\bar{\gamma}$ at the $1 \%$ level].

Besides the "Nordtvedt effect" proper (i.e. the effect of $\bar{\delta}_{12} \neq 0$ ), that we discuss here, there are some other null effects which are more sensitive to $\bar{\beta}$ and $\bar{\gamma}$ than the non-Einsteinian modifications of non-null general relativistic effects. A subdominant null effect comes from the violation of the equivalence principle associated with the gravitational binding energy 
of the Earth-Moon system. In lowest approximation (linear in $m$ ), it is equivalent (see e.g. Eq. (3.14b) of Ref. [34]) to replacing $\bar{\delta}_{12}$ by

$$
\tilde{\delta}_{12}=\bar{\delta}_{12}-\frac{1}{3} \eta\left(\frac{n a}{c}\right)^{2}
$$

where $a$ denotes the semi-major axis of the lunar orbit and where $\eta$ denotes, as usual, the combination

$$
\eta \equiv 4 \bar{\beta}-\bar{\gamma}=4 \beta-\gamma-3
$$

In general, $\bar{\delta}_{12}$ is the sum of two physically independent contributions

$$
\bar{\delta}_{12}=\left(\hat{\delta}_{1}-\hat{\delta}_{2}\right)+\eta\left(\frac{E_{1}^{\text {grav }}}{m_{1} c^{2}}-\frac{E_{2}^{\text {grav }}}{m_{2} c^{2}}\right) .
$$

The first contribution $\hat{\delta}_{12} \equiv \hat{\delta}_{1}-\hat{\delta}_{2}$ is generically expected to be present because the best motivated modified theories of gravity violate the "weak equivalence principle", i.e. contain, besides Einstein's universal tensor interaction, some composition-dependent couplings that make laboratory bodies fall in a non universal way (see e.g. [35], [36]). The second contribution on the right-hand side of Eq. (2.3) (proportional to $\eta$ like the correction in Eq. (2.1)) contains the gravitational self-energy of the bodies $(A=1,2)$

$$
E_{A}^{\text {grav }}=-(G / 2) \int_{A} \int_{A} d^{3} x d^{3} x^{\prime} \rho(\mathbf{x}) \rho\left(\mathbf{x}^{\prime}\right) /\left|\mathbf{x}-\mathbf{x}^{\prime}\right|
$$

and is the one first pointed out by Nordtvedt [11], [12]. As indicated by Dicke [13], [14], it is present in all gravity theories where the effective, locally measured gravitational "constant" may vary from place to place (see e.g. Section V B of [35]). We shall take as nominal values for the gravitational self-energies of the Moon (label 1) and the Earth (label 2) the values adopted by Williams, Newhall and Dickey [33], namely $E_{1}^{\text {grav }} / m_{1} c^{2}=-0.19 \times 10^{-10}$, $E_{2}^{\text {grav }} / m_{2} c^{2}=-4.64 \times 10^{-10}$, so that

$$
\frac{E_{1}^{\text {grav }}}{m_{1} c^{2}}-\frac{E_{2}^{\text {grav }}}{m_{2} c^{2}}=4.45 \times 10^{-10} .
$$

We then find numerically that the modification due to the gravitational binding energy of the Earth-Moon system, $-\frac{1}{3} \eta n^{2} a^{2} / c^{2}=-\frac{1}{3} \eta G\left(m_{1}+m_{2}\right) / a c^{2}$ in Eq. (2.1), is (to first order) equivalent to decreasing the nominal value $(2.5)$ by $-0.039 \times 10^{-10}$. This represents a fractional change of 2.5$)$ by $-0.87 \%$ which is probably smaller than the uncertainty in the estimate (2.5) associated with our imperfect knowledge of the internal structures of the Earth and the Moon. These orders of magnitude illustrate the fact that the overwhelmingly dominant sensitivity of the lunar motion to non-Einsteinian effects comes from the terms proportional to $\bar{\delta}_{12}$ that we concentrate upon in the following.

\section{B. Three-body Lagrangian}

The Lagrangian describing the $N$-body problem in the currently best motivated relativistic theories of gravity, i.e. those where gravity is mediated both by a tensor field and 
a scalar field with, generically, composition-dependent couplings (see e.g. [35], [36]), can be written as

$$
L_{\bar{\beta}, \bar{\gamma}, \bar{\delta}}=L_{G R}+L_{\bar{\beta}}+L_{\bar{\gamma}}+L_{\bar{\delta}}
$$

where $L_{G R}$ denotes the general relativistic contribution (in which one should use an effective value of the gravitational coupling constant $G$ which incorporates the compositionindependent part of the interaction mediated by the scalar field),

$$
L_{\bar{\gamma}}=\frac{1}{2 c^{2}} \bar{\gamma} \sum_{A \neq B} \frac{G m_{A} m_{B}}{r_{A B}}\left(\mathbf{v}_{A}-\mathbf{v}_{B}\right)^{2},
$$

denotes the (non-tensor-like) velocity-dependent part of the two-body scalar interaction (one-scalaron exchange level),

$$
L_{\bar{\beta}}=-\frac{1}{c^{2}} \bar{\beta} \sum_{B \neq A \neq C} \frac{G^{2} m_{A} m_{B} m_{C}}{r_{A B} r_{A C}},
$$

denotes the modification of the non-linear three-body general relativistic interaction due to the scalar interaction, and

$$
L_{\bar{\delta}}=\frac{1}{2} \sum_{A \neq B}\left(\bar{\delta}_{A}+\bar{\delta}_{B}\right) \frac{G m_{A} m_{B}}{r_{A B}},
$$

with

$$
\bar{\delta}_{A}=\hat{\delta}_{A}+\eta \frac{E_{A}^{\text {grav }}}{m_{A} c^{2}}
$$

represents (to lowest order) the combined effect of the composition-dependent couplings $\left(\hat{\delta}_{A} \neq 0\right.$; violation of the "weak equivalence principle") and of the Dicke-Nordtvedt contribution due to the spatial variability of the effective gravitational coupling constant ( $\eta=4 \bar{\beta}-\bar{\gamma} \neq 0$; violation of the "strong equivalence principle"). For a direct, field-theory derivation of $L_{\bar{\gamma}}$ and $L_{\bar{\beta}}$ and the expression of the phenomenological Eddington parameters $\bar{\gamma}=\gamma-1$ and $\bar{\beta}=\beta-1$ in terms of the basic coupling parameters of the scalar field (as well as the generalization of these results to the case of strongly self-gravitating bodies) see Ref. [37].

We assume here that all the general relativistic contributions to the lunar motion (and to its observation through laser ranging) are separately worked out with sufficient accuracy, using, for instance, the new, complete framework for relativistic celestial mechanics of Ref. 38 (which provides the first consistent relativistic description of the multipole moments of extended bodies). Following the discussion above, we henceforth discard the subdominant contributions coming from $L_{\bar{\beta}}$ and $L_{\bar{\gamma}}$ to concentrate upon the effects due to $L_{\bar{\delta}}$. [The barycentric frame contributions of $L_{\bar{\beta}}$ and $L_{\bar{\gamma}}$ have been accurately computed by Brumberg and Ivanova [18] and can be linearly superposed with the ones of $L_{\bar{\delta}}$.] Finally, it is enough to consider the sum of the lowest-order approximation to $L_{G R}$ and of $L_{\bar{\delta}}$, namely 


$$
L\left(\mathbf{x}_{A}, \mathbf{v}_{A}\right)=\sum_{A} \frac{1}{2} m_{A} \mathbf{v}_{A}^{2}+\frac{1}{2} \sum_{A \neq B} \frac{G_{A B} m_{A} m_{B}}{r_{A B}}
$$

where

$$
G_{A B}=G\left[1+\bar{\delta}_{A}+\bar{\delta}_{B}\right]
$$

with $\bar{\delta}_{A}$ of the form given in (2.10), represents the effective, composition-dependent gravitational coupling between the massive bodies $A$ and $B$. In Eq. (2.11) $\mathbf{v}_{A}=d \mathbf{x}_{A} / d t$ denotes the (barycentric) velocity of body $A, m_{A}$ the (inertial) mass of $A$ and $r_{A B}=\left|\mathbf{x}_{A}-\mathbf{x}_{B}\right|$ the distance between $A$ and $B$. We consider a three-body problem, and more particularly the Moon-Earth-Sun system ( $1=$ Moon, $2=$ Earth, $3=$ Sun). Evidently, all our analytical results will apply if 2 denotes another planet, and 1 one of its natural or artificial satellites. [Note, however, that the relative orders of magnitude of the non-Einsteinian effects is different for low-orbit artificial Earth satellites. See [34] for a recent discussion.]

Starting from Eq. (2.11), we first separate the variables describing the motion of the center of mass of the Earth-Moon system,

$$
\begin{aligned}
m_{0} \mathbf{x}_{0} & \equiv m_{1} \mathbf{x}_{1}+m_{2} \mathbf{x}_{2} \\
m_{0} & \equiv m_{1}+m_{2}
\end{aligned}
$$

and $\mathbf{v}_{0} \equiv d \mathbf{x}_{0} / d t$, from those describing the relative lunar motion,

$$
\begin{aligned}
\mathbf{x}_{12} & \equiv \mathbf{x}_{1}-\mathbf{x}_{2}, \\
\mu_{12} & \equiv \frac{m_{1} m_{2}}{m_{1}+m_{2}},
\end{aligned}
$$

and $\mathbf{v}_{12} \equiv d \mathbf{x}_{12} / d t$. This yields

$L\left(\mathbf{x}_{0}, \mathbf{x}_{12}, \mathbf{x}_{3} ; \mathbf{v}_{0}, \mathbf{v}_{12}, \mathbf{v}_{3}\right)=\frac{1}{2} m_{0} \mathbf{v}_{0}^{2}+\frac{1}{2} \mu_{12} \mathbf{v}_{12}^{2}+\frac{1}{2} m_{3} \mathbf{v}_{3}^{2}+G_{12} \frac{m_{1} m_{2}}{r_{12}}+G_{13} \frac{m_{1} m_{3}}{r_{13}}+G_{23} \frac{m_{2} m_{3}}{r_{23}}$,

where

$$
\begin{aligned}
& r_{13}=\left|\mathbf{x}_{3}-\mathbf{x}_{0}-X_{2} \mathbf{x}_{12}\right| \\
& r_{23}=\left|\mathbf{x}_{3}-\mathbf{x}_{0}+X_{1} \mathbf{x}_{12}\right| \\
& X_{1} \equiv m_{1} / m_{0}, \quad X_{2} \equiv m_{2} / m_{0}=1-X_{1} .
\end{aligned}
$$

Expanding $r_{13}^{-1}$ and $r_{23}^{-1}$ in powers of $r_{12} / r_{30}\left(r_{30}=\left|\mathbf{x}_{30}\right|\right.$ with $\left.\mathbf{x}_{30}=\mathbf{x}_{3}-\mathbf{x}_{0}\right)$ leads to

$$
\begin{aligned}
L & =L_{03}\left(\mathbf{x}_{0}, \mathbf{x}_{3}, \mathbf{v}_{0}, \mathbf{v}_{3}\right)+\mu_{12} \hat{L}_{12}\left(\mathbf{x}_{12}, \mathbf{v}_{12}, \mathbf{x}_{30}\right), \\
L_{03} & =\frac{1}{2} m_{0} \mathbf{v}_{0}^{2}+\frac{1}{2} m_{3} \mathbf{v}_{3}^{2}+\left(X_{1} G_{13}+X_{2} G_{23}\right) \frac{m_{0} m_{3}}{r_{03}}, \\
\hat{L}_{12} & =\frac{1}{2} \mathbf{v}_{12}^{2}+G_{12} \frac{m_{0}}{r_{12}}+R_{1}+R_{2}+R_{3}+\cdots,
\end{aligned}
$$




$$
\begin{aligned}
& R_{1}=m_{3}\left(-G_{13}+G_{23}\right) x_{12}^{i} \partial_{i}^{(3)} \frac{1}{r_{30}}, \\
& R_{2}=\frac{1}{2 !} m_{3}\left(G_{13} X_{2}+G_{23} X_{1}\right) x_{12}^{i} x_{12}^{j} \partial_{i j}^{(3)} \frac{1}{r_{30}}, \\
& R_{3}=\frac{1}{3 !} m_{3}\left(-G_{13} X_{2}^{2}+G_{23} X_{1}^{2}\right) x_{12}^{i} x_{12}^{j} x_{12}^{k} \partial_{i j k}^{(3)} \frac{1}{r_{30}},
\end{aligned}
$$

where $\partial_{i}^{(3)} \equiv \partial / \partial x_{3}^{i}, \partial_{i j}^{(3)} \equiv \partial^{2} / \partial x_{3}^{i} \partial x_{3}^{j}, \ldots$ Note that the suffices $1,2,3, \ldots$ in $R_{n}$ 's have nothing to do with the body labels $A, B=1,2,3$, but keep track of the successive powers of $\mathbf{x}_{12}$.

To a very good approximation we can consider that, in the (normalized) Earth-Moon Lagrangian (2.21), the motion of the Sun with respect to the Earth-Moon barycenter, $\mathbf{x}_{30}(t)$, is obtained by solving the two-body Lagrangian (2.20). After separating in Eq. (2.20) the motion of the center of mass of the Earth-Moon-Sun system, the reduced Lagrangian describing the dynamics of the relative motion $\mathbf{x}_{30}$ is

$$
\hat{L}_{03}^{\text {relative }}=\frac{1}{2} \mathbf{v}_{03}^{2}+G_{03} \frac{m_{0}+m_{3}}{r_{03}},
$$

where we introduced

$$
G_{03} \equiv X_{1} G_{13}+X_{2} G_{23} \text {. }
$$

Therefore, seen from the Earth-Moon barycenter, the Sun undergoes a Keplerian, elliptic motion corresponding to a total effective gravitational mass $G_{03}\left(m_{0}+m_{3}\right)=G_{03}\left(m_{1}+m_{2}+\right.$ $m_{3}$ ). If we denote (as is traditional in lunar theory) the mean angular velocity and the semi-major axis of this elliptic motion as $n^{\prime}$ and $a^{\prime}$, respectively, we can write

$$
n^{\prime 2} a^{\prime 3}=G_{03}\left(m_{0}+m_{3}\right)=G_{03}\left(m_{1}+m_{2}+m_{3}\right) .
$$

For simplicity's sake, we shall in fact consider the "Main Problem" of lunar theory in which the Sun is considered as moving in a circle of radius $a^{\prime}$ with the constant angular velocity $n^{\prime}$.

Evaluating the derivatives with respect to $\mathbf{x}_{3}$ in Eqs. (2.22), and using Eq. (2.12), yields

$$
\begin{aligned}
& R_{1}=m_{3}^{\text {grav }} \bar{\delta}_{12} \frac{\mathbf{N} . \mathbf{r}}{r^{\prime 2}} \\
& R_{2}=m_{3}^{\text {grav }}\left[1+\left(X_{2}-X_{1}\right) \bar{\delta}_{12}\right] \frac{3(\mathbf{N} . \mathbf{r})^{2}-\mathbf{r}^{2}}{2 r^{\prime 3}} \\
& R_{3} \simeq m_{3}^{\text {grav }}\left(X_{2}-X_{1}\right) \frac{5(\mathbf{N} . \mathbf{r})^{3}-3(\mathbf{N} \cdot \mathbf{r}) \mathbf{r}^{2}}{2 r^{\prime 4}}
\end{aligned}
$$

To simplify the notation, we have written $\mathbf{r} \equiv \mathbf{x}_{12}=\mathbf{x}_{1}-\mathbf{x}_{2}, \mathbf{N} \equiv \mathbf{x}_{30} / r_{30}$ (directed toward the Sun), $r^{\prime} \equiv r_{30}, m_{3}^{\text {grav }} \equiv G_{03} m_{3}$, where we recall that $G_{03}$ is the weighted average (2.24), and

$$
\bar{\delta}_{12} \equiv \bar{\delta}_{1}-\bar{\delta}_{2}
$$

which agrees with our previous expressions (1.1) or (2.3). In the (much smaller) term $R_{3}$ we have neglected the $\bar{\delta}$-modifications. 


\section{Hill's equations of motion}

Following Euler's second lunar theory and Hill [39], [40], it is convenient to refer the motion of the Moon to axes rotating with the mean angular velocity of the Sun. For simplicity, we shall consider the main lunar problem in which (i) the Sun is considered as moving in a circle of radius $a^{\prime}$ with the uniform angular velocity $n^{\prime}$, (ii) the Moon moves in the same plane as the Sun, and (iii) one looks for a periodic motion of the Moon in the frame rotating with the angular velocity $n^{\prime}$. Taking into account the lunar and solar eccentricities $e$ and $e^{\prime}$, and the lunar inclination $I$, is expected to modify our results by terms of order $O\left(e^{2}, e^{2}, \sin ^{2} I\right) \lesssim 1 \%$.

With respect to the rotating frame $\left(\mathbf{e}_{X}(t), \mathbf{e}_{Y}(t)\right)$, with $\mathbf{e}_{X}=\mathbf{N}$ directed toward the Sun, the position and velocity vectors of the Moon read $\mathbf{r}=\mathbf{x}_{12}=X \mathbf{e}_{X}+Y \mathbf{e}_{Y}, \mathbf{v}=\mathbf{v}_{12}=$ $\left(\dot{X}-n^{\prime} Y\right) \mathbf{e}_{X}+\left(\dot{Y}+n^{\prime} X\right) \mathbf{e}_{Y}$ (the overdot denoting $\left.d / d t\right)$. When expanding the kinetic terms in the reduced, relative Lagrangian (2.21), i.e. $\frac{1}{2} \mathbf{v}^{2}=\frac{1}{2}\left(\dot{X}-n^{\prime} Y\right)^{2}+\frac{1}{2}\left(\dot{Y}+n^{\prime} X\right)^{2}$, one recognizes the usual Coriolis (terms linear in $\dot{X}$ and $\dot{Y}$ ) and centrifugal (terms quadratic in $X$ and $Y$ ) effects. The centrifugal terms can be gathered with the contribution $R_{2}$, Eq. (2.26b), which is also quadratic in $X$ and $Y$. The resulting Lagrangian describing the dynamics in the rotating frame reads

$$
\hat{L}_{12}(X, Y, \dot{X}, \dot{Y})=\frac{1}{2}\left(\dot{X}^{2}+\dot{Y}^{2}\right)+n^{\prime}(X \dot{Y}-Y \dot{X})+F(X, Y) .
$$

Here, $F(X, Y)=G_{12} m_{0} / r+R_{1}+\left[R_{2}+\frac{1}{2} n^{\prime 2}\left(X^{2}+Y^{2}\right)\right]+R_{3}+\cdots=F_{0}+F_{1}+F_{2}+F_{3}+\cdots$ is a time-independent potential with

$$
\begin{aligned}
& F_{0}=\frac{G_{12} m_{0}}{\sqrt{X^{2}+Y^{2}}} \\
& F_{1}=\frac{n^{\prime 2} a^{\prime}}{1+m_{0} / m_{3}} \bar{\delta}_{12} X \\
& F_{2}=\frac{1}{2} \frac{n^{\prime 2}}{1+m_{0} / m_{3}}\left\{\left[1+\left(X_{2}-X_{1}\right) \bar{\delta}_{12}\right]\left(3 X^{2}\right)+\left[\frac{m_{0}}{m_{3}}-\left(X_{2}-X_{1}\right) \bar{\delta}_{12}\right]\left(X^{2}+Y^{2}\right)\right\} \\
& F_{3} \simeq \frac{1}{2} \frac{n^{\prime 2}}{a^{\prime}}\left(X_{2}-X_{1}\right)\left[5 X^{3}-3 X\left(X^{2}+Y^{2}\right)\right] .
\end{aligned}
$$

In writing out Eqs. (2.29) we have replaced $m_{3}^{\text {grav }}=G_{03} m_{3}$ by the expression $m_{3}^{\text {grav }}=$ $n^{\prime 2} a^{\prime 3} /\left(1+m_{0} / m_{3}\right)$ obtained from Eq. (2.25). The equations of motion corresponding to the Lagrangian (2.28) read

$$
\begin{aligned}
& \frac{d^{2} X}{d t^{2}}-2 n^{\prime} \frac{d Y}{d t}=\frac{\partial F}{\partial X} \\
& \frac{d^{2} Y}{d t^{2}}+2 n^{\prime} \frac{d X}{d t}=\frac{\partial F}{\partial Y}
\end{aligned}
$$

We see from Eqs. (2.29) that a violation of the equivalence principle has several consequences in the lunar theory: (i) the effective gravitational constant appearing in front of the Earth-Moon total mass $m_{0}=m_{1}+m_{2}$, namely $G_{12}=G\left(1+\bar{\delta}_{1}+\bar{\delta}_{2}\right)$, differs from the one 
appearing in the theory of the Earth orbital motion, $G_{03}$ defined in Eq. (2.24); (ii) there is a new term, linear in $X$, in the Lagrangian, $F_{1}$, Eq. 2.29b); (iii) the usual tidal plus centrifugal potential $F_{2}$ (as well as the higher-order tidal potentials) is fractionally modified by $\bar{\delta}_{12} \neq 0$. The effect (i) has practically no observational consequences as, for instance, the "GM" of the Earth is measured much more accurately from Earth satellites (artificial or natural) than from the correction it brings in the Earth-Sun interaction. The effect (ii) is the one discussed by Newton, Laplace and Nordtvedt, that we shall study in detail below. As for the effects (iii) it will be clear from the following that they are numerically negligible compared to the effects of $F_{1}$ because the corresponding source terms in the equations of motion are smaller by a factor $r / a^{\prime} \simeq 1 / 400$, and, moreover, the corresponding solution is not amplified (as the $F_{1}$-effects) by a small divisor $1 / m \simeq 12$ because they correspond to the driving frequency $2\left(n-n^{\prime}\right)$ (instead of $n-n^{\prime}$ for $F_{1}$ ). Finally, as we can also neglect the fractional correction $\left(1+m_{0} / m_{3}\right)^{-1}$ to $\bar{\delta}_{12}$ in (2.29b) $\left[m_{0} / m_{3} \simeq 1 / 328900\right]$, we shall keep Eqs. (2.29a) and (2.29d ) and replace (2.29b), (2.29d) by

$$
\begin{aligned}
& F_{1} \simeq n^{\prime 2} a^{\prime} \bar{\delta}_{12} X, \\
& F_{2} \simeq \frac{3}{2} n^{\prime 2} X^{2} .
\end{aligned}
$$

The contribution $F_{3}$ to the potential $F$ (octupolar tide) generates the so-called "parallactic" terms in the lunar motion. Compared to the usual (quadrupolar) tidal contribution, they contain the small parameter $r / a^{\prime} \simeq 1 / 400$. Hill's approach consists of solving first exactly (in the sense of infinite power series) the dynamics defined by the truncated potential $F_{\text {Hill }}=F_{0}+F_{2}=G_{12} m_{0} / r+\frac{3}{2} n^{\prime 2} X^{2}$. [The parallactic terms are obtained later by perturbing Hill's main problem.] In presence of a violation of the equivalence problem we have to add the term $F_{1}$, Eq. (2.31a), to $F_{\text {Hill }}$ ("dipolar tide"!). The resulting equations of motion (2.30) read explicitly

$$
\begin{aligned}
\frac{d^{2} X}{d t^{2}}-2 n^{\prime} \frac{d Y}{d t}= & -\frac{G_{12} m_{0}}{r^{3}} X \\
& +3 n^{\prime 2} X+n^{\prime 2} a^{\prime} \bar{\delta}_{12}, \\
\frac{d^{2} Y}{d t^{2}}+2 n^{\prime} \frac{d X}{d t}= & -\frac{G_{12} m_{0}}{r^{3}} Y .
\end{aligned}
$$

They admit (in the general case of a time-independent potential $F$ ) the Jacobi energy integral

$$
\frac{1}{2}\left(\dot{X}^{2}+\dot{Y}^{2}\right)-F(X, Y)=C=\text { const. }
$$

\section{Iterative solution of Hill's equations}

In spite of the apparent simplicity of Eqs. (2.32) and of the existence of the first integral (2.33), the corresponding dynamics contains all the richness and complexity of the threebody problem. Hill's idea was first to find an exact periodic solution of Eqs. (2.32) (with $\bar{\delta}_{12}=0$ ). The existence (in a mathematical sense) of Hill's periodic solution, and the 
convergence of the power series (in the parameter $\left.m \equiv n^{\prime} /\left(n-n^{\prime}\right)\right)$ giving its explicit form, have been proven by Wintner [41] (see [42] for more references). The existence of such a one-parameter family of coplanar, periodic solutions, and the convergence of the associated perturbation series in $m$, are stable under the addition of the full series of higher-order tidal terms [43. We are therefore confident that it will still exist when one adds the "dipole tidal" term $F_{1}$, if $\bar{\delta}_{12}$ is small enough.

To construct explicitly the perturbation series in $m$ giving the periodic solutions of Eqs. (2.32) it is convenient to rewrite them in terms of new variables. Following a standard notation [17] (except that we do not introduce a separate letter for the complex conjugate of $u$ ) we define

$$
\begin{aligned}
u & =X+i Y \\
\bar{u} & =X-i Y \\
\tau & =\left(n-n^{\prime}\right) t+\tau_{0} \\
\zeta & =e^{i \tau} \\
D & =\frac{1}{i} \frac{d}{d \tau}=\zeta \frac{d}{d \zeta}
\end{aligned}
$$

Here, $n$ denotes the mean sidereal orbital velocity of the periodic solution one is looking for (in other words the rotating frame quantities $X, Y, u, \bar{u}$ are supposed to be periodic functions of $\tau$ with period $2 \pi)$. The parameter $m=n^{\prime} /\left(n-n^{\prime}\right)$ is the remaining free parameter of the problem. It takes positive values for prograde orbits (going in the same sense as the Sun': $0<n^{\prime}<n$ ), and negative values for retrograde orbits $n<0$. With this notation the general equations of motion (2.30) read

$$
D^{2} u+2 m D u=-2 \frac{\partial \hat{F}}{\partial \bar{u}}
$$

(and its complex conjugate: $u \rightarrow \bar{u}, D \rightarrow \bar{D}=-D$ ) where $\hat{F} \equiv\left(n-n^{\prime}\right)^{-2} F=m^{2} n^{\prime-2} F$. For small values of $m$ (i.e. orbits of small radius around the Earth) the periodic solution of Eq. (2.35) is of the form $u \simeq$ (const.) $\zeta, \bar{u} \simeq$ (const.) $\bar{\zeta}=$ (const.) $\zeta^{-1}$. It is convenient to replace $u$ by a variable $w$ which tends to zero with $m$. Following Liapunov 44 and Brumberg and Ivanova [18, one defines first a fiducial lunar semi-major axis $\tilde{a}$ by writing

$$
\frac{G_{12} m_{0}}{\left(n-n^{\prime}\right)^{2} \tilde{a}^{3}}=\kappa(m),
$$

where

$$
\kappa(m) \equiv 1+2 m+\frac{3}{2} m^{2}
$$

With this definition of $\tilde{a}$ one introduces the variable $w$ by

\footnotetext{
${ }^{3}$ We shall not consider the prograde orbits with $0<n<n^{\prime}$ which are highly unstable; see below.
} 


$$
\begin{aligned}
& u=\tilde{a} \zeta(1+w), \\
& \bar{u}=\tilde{a} \zeta^{-1}(1+\bar{w}) .
\end{aligned}
$$

The Lagrangian $\mathcal{L}=-2 \tilde{a}^{-2}\left(n-n^{\prime}\right)^{-2} \hat{L}_{12}$ can be written as

$$
\begin{aligned}
\mathcal{L}(w, \bar{w}, D w, D \bar{w})= & D w D \bar{w}+2(m+1) w D \bar{w} \\
& -G(w, \bar{w})+(\text { total derivative })
\end{aligned}
$$

where $G \equiv 2 \tilde{a}^{-2} \hat{F}+(1+2 m)(1+w)(1+\bar{w})$, and the associated equations of motion read

$$
D^{2} w+2(m+1) D w=-\frac{\partial G}{\partial \bar{w}} .
$$

The explicit form of the potential $G(w, \bar{w})$ in our problem (i.e. when $\left(n-n^{\prime}\right)^{2} \hat{F} \equiv F=$ $\left.F_{0}+F_{1}+F_{2}\right)$ reads

$$
\begin{aligned}
G(w, \bar{w})= & \kappa(m)\left[2(1+w)^{-1 / 2}(1+\bar{w})^{-1 / 2}+(1+w)(1+\bar{w})\right]+\hat{\lambda}\left[\zeta(1+w)+\zeta^{-1}(1+\bar{w})\right] \\
& +\frac{3}{4} m^{2}\left[\zeta^{2}(1+w)^{2}+\zeta^{-2}(1+\bar{w})^{2}\right]
\end{aligned}
$$

where we have introduced, instead of $\bar{\delta}_{12}$, the small dimensionless parameter

$$
\hat{\lambda} \equiv m^{2} \bar{\delta}_{12} \frac{a^{\prime}}{\tilde{a}}
$$

The corresponding equations of motion read

$$
D^{2} w+2(m+1) D w+\kappa\left[1+w-(1+w)^{-1 / 2}(1+\bar{w})^{-3 / 2}\right]+\hat{\lambda} \zeta^{-1}+\frac{3}{2} m^{2} \zeta^{-2}(1+\bar{w})=0 .
$$

A last transformation [44], [18] consists of separating off the square bracket multiplied by $\kappa$ in (2.43) its nonlinear piece, namely

$Q(w, \bar{w}) \equiv(1+w)^{-1 / 2}(1+\bar{w})^{-3 / 2}-1+\frac{1}{2} w+\frac{3}{2} \bar{w}=\frac{3}{8} w^{2}+\frac{15}{8} \bar{w}^{2}+\frac{3}{4} w \bar{w}+\mathcal{O}\left(w^{3}, w^{2} \bar{w}, w \bar{w}^{2}, \bar{w}^{3}\right)$

We can now define a linear operator acting on $(w, \bar{w})$,

$$
L(w, \bar{w}) \equiv D^{2} w+2(m+1) D w+\frac{3}{2} \kappa(m)(w+\bar{w})
$$

and an effective source term (containing source terms and non-linearities)

$$
W(w, \bar{w}) \equiv-\hat{\lambda} \zeta^{-1}-\frac{3}{2} m^{2} \zeta^{-2}(1+\bar{w})+\kappa(m) Q(w, \bar{w}) .
$$

In terms of these definitions the equations of motion read 


$$
L(w, \bar{w})=W(w, \bar{w})
$$

and its complex conjugate equation. This is the form used by Brumberg and Ivanova 18 in their study of relativistic effects in the lunar motion.

Note that the source of all equivalence-principle violation effects is the contribution $-\hat{\lambda} \zeta^{-1}$ on the right-hand side of Eq. (2.46), with $\hat{\lambda}$ defined by Eq. (2.42). Even when restricting oneself (as we shall) to the effects linear in $\hat{\lambda}$, the corresponding contributions in the solution are quite complicated because of the interplay with the quadrupole tidal effects (from $F_{2}$ ), i.e., mathematically, because of the mixed term $-\frac{3}{2} m^{2} \zeta^{-2} \bar{w}$ and the nonlinear term $\propto Q(w, \bar{w})$ in $W(w, \bar{w})$.

When putting back the octupole tidal effects $\left(F_{3}\right.$, Eq. (2.29d)) they add to the effective source term (2.46)) the contribution

$$
W_{3}(w, \bar{w})=-\frac{3}{8} \hat{\pi}\left[\zeta(1+w)^{2}+2 \zeta^{-1}(1+w)(1+\bar{w})+5 \zeta^{-3}(1+\bar{w})^{2}\right]
$$

where

$$
\hat{\pi} \equiv m^{2}\left(X_{2}-X_{1}\right) \frac{\tilde{a}}{a^{\prime}}=m^{2} \frac{m_{2}-m_{1}}{m_{2}+m_{1}} \frac{\tilde{a}}{a^{\prime}} .
$$

The equation (2.47) can be solved by iteration: first, one keeps only the linear source terms which exist when $w=0$, namely $W^{(1)}\left(\zeta, \zeta^{-1}\right)=W(0,0)=-\hat{\lambda} \zeta^{-1}-\frac{3}{2} m^{2} \zeta^{-2}$ [with the addition of $W_{3}(0,0)=-\frac{3}{8} \hat{\pi}\left(\zeta+2 \zeta^{-1}+5 \zeta^{-3}\right)$ when including parallactic terms]. Second, one solves the linear equations $L(w, \bar{w})=W^{(1)}\left(\zeta, \zeta^{-1}\right)$ to get the corresponding first-order solution: $w^{(1)}=\hat{\lambda} w_{\lambda}^{(1)}+m^{2} w_{m^{2}}^{(1)}+\hat{\pi} w_{\pi}^{(1)}$, which is valid up to terms of higher order in the formal expansion parameters $\hat{\lambda}, m^{2}$ and $\hat{\pi}$. The next step is to insert the first-order solution $w^{(1)}$ in the full source term $W(w, \hat{w})$ and to collect the second-order source term $W^{(2)}\left(\zeta, \zeta^{-1}\right)$ of formal order $\left(\hat{\lambda}+m^{2}+\hat{\pi}\right)^{2}$. The corresponding second-order solution $w^{(2)}$ is obtained by solving the linear equation $L\left(w^{(2)}, \bar{w}^{(2)}\right)=W^{(2)}\left(\zeta, \zeta^{-1}\right)$, etc. At each stage of the iteration, one deals with a source term which is a linear combination (with real coefficients of a finite number of integer powers of $\zeta$ and $\zeta^{-1}$, say

$$
W_{\star}=W_{0}+W_{1} \zeta+W_{-1} \zeta^{-1}+W_{2} \zeta^{2}+W_{-2} \zeta^{-2}+\cdots+W_{k} \zeta^{k}+W_{-k} \zeta^{-k} .
$$

It is easy to check that there is a unique solution of the linear system $L(w, \bar{w})=W_{\star}$, $\bar{L}(w, \bar{w})=\bar{W}_{\star}$ and that it is given by

$$
w_{\star}=w_{0}+w_{1} \zeta+w_{-1} \zeta^{-1}+w_{2} \zeta^{2}+w_{-2} \zeta^{-2}+\cdots+w_{k} \zeta^{k}+w_{-k} \zeta^{-k},
$$

with 18

\footnotetext{
${ }^{4}$ The reality of all coefficients $W_{k}$ in Eq. (2.50) and $w_{k}$ in Eq. (2.51) is easily proven by induction, given the reality of the coefficients in the exact definition of $W(w, \bar{w})$ and in the iterative solution Eq. (2.52).
} 


$$
\begin{aligned}
w_{0} & =\frac{1}{3 \kappa(m)} W_{0}, \\
w_{k} & =\frac{1}{\Delta_{k}}\left[\left(k^{2}-2(m+1) k+\frac{3}{2} \kappa\right) W_{k}-\frac{3}{2} \kappa W_{-k}\right], \\
w_{-k} & =\frac{1}{\Delta_{k}}\left[\left(k^{2}+2(m+1) k+\frac{3}{2} \kappa\right) W_{-k}-\frac{3}{2} \kappa W_{k}\right],
\end{aligned}
$$

Here $\Delta_{k}\left(=\Delta_{-k}\right)$ denotes the determinant of the $2 \times 2$ system of equations satisfied by $w_{k}$ and $w_{-k}($ when $k \neq 0)$. Its value is

$$
\Delta_{k}=k^{2}\left[k^{2}+3 \kappa-4(m+1)^{2}\right]=k^{2}\left[k^{2}-1-2 m+\frac{1}{2} m^{2}\right] .
$$

This determinant never vanishes $(k \neq 0)$, but it takes a small value of order $m$ when $k= \pm 1$. This small divisor is one of the origins of the peculiar amplification which affects both equivalence-principle-violation effects $\left(W^{(1)}\left(F_{1}\right)=-\hat{\lambda} \zeta^{-1}\right)$ and a part of the octupolar-tide effects $\left(W^{(1)}\left(F_{3}\right)=-\frac{3}{8} \hat{\pi}\left(\zeta+2 \zeta^{-1}+\cdots\right)\right)$. A consequence of the small divisor $\Delta_{1}=-2 m+\frac{1}{2} m^{2}$ is that, when collecting from the iterative solution the contributions proportional to $\hat{\lambda} \zeta^{ \pm 1}$ and $\hat{\pi} \zeta^{ \pm 1}$ they are found to proceed according to the powers of $m^{2} / \Delta_{1}=\mathcal{O}(m)$ instead of the powers of $m^{2}$ as formally expected from the structure (2.46)

The first steps of the iteration can be done by hand. From Eqs. (2.52) the linearized solution (without parallactic terms) has the form

$$
w^{(1)}=w_{1}^{(1)} \zeta+w_{-1}^{(1)} \zeta^{-1}+w_{2}^{(1)} \zeta^{2}+w_{-2}^{(1)} \zeta^{-2},
$$

with

$$
\begin{aligned}
w_{1}^{(1)} & =+\frac{3}{2} \frac{\kappa}{\Delta_{1}} \hat{\lambda}, \\
w_{-1}^{(1)} & =-\frac{1+2(m+1)+\frac{3}{2} \kappa}{\Delta_{1}} \hat{\lambda}, \\
w_{2}^{(1)} & =+\frac{9}{4} \frac{\kappa}{\Delta_{2}} m^{2}, \\
w_{-2}^{(1)} & =-\frac{3}{2} \frac{4+4(m+1)+\frac{3}{2} \kappa}{\Delta_{2}} m^{2} .
\end{aligned}
$$

Here, as defined above, $\kappa \equiv 1+2 m+\frac{3}{2} m^{2}, \Delta_{1}=-2 m+\frac{1}{2} m^{2}$ and $\Delta_{2}=4\left(3-2 m+\frac{1}{2} m^{2}\right)$. The insertion of the linearized solution $w^{(1)}$, Eqs. (2.55), into $W(w, \bar{w})$, Eq. (2.46), generates a second-order source term with the following structure: $W^{(2)} \sim \hat{\lambda} m^{2}\left(\zeta^{ \pm 1}+\zeta^{ \pm 3}\right)+m^{4}\left(\zeta^{0}+\zeta^{ \pm 2}+\right.$ $\left.\zeta^{ \pm 4}\right)+\mathcal{O}\left(\hat{\lambda}^{2}\right)$. Let us focus on the terms in the solution which are linear in $\hat{\lambda}$ and contain the "resonant" frequencies $\zeta^{ \pm 1}$. Their source terms are found to be $W_{1}^{(2)}=\frac{99}{64} m \hat{\lambda}(1+\mathcal{O}(m))$ and

\footnotetext{
${ }^{5}$ Following Poincaré [45] (see also Ref. 17]), one can clarify the iterative process by giving a new name to the parameter $m$ appearing in the second term of $W$, Eq. (2.46), leaving unchanged the other occurrences of $m$ in $L$ and $\kappa(m)$.
} 
$W_{-1}^{(2)}=-\frac{207}{64} m \hat{\lambda}(1+\mathcal{O}(m))$. From Eqs. (2.52) the corresponding solutions can be written as $w_{1}^{(2)}=-\frac{45}{16} \hat{\lambda}(1+\mathcal{O}(m)), w_{-1}^{(2)}=-3 w_{1}^{(2)}(1+\mathcal{O}(m))$. At this approximation we have, when expanding $w_{ \pm 1}^{(1)}$ in powers of $m$,

$$
\begin{aligned}
w_{1}^{(1)}+w_{1}^{(2)} & =\left(1+\frac{15}{4} m+\mathcal{O}\left(m^{2}\right)\right) w_{1}^{(1)} \\
& =-\frac{3}{4} \frac{\hat{\lambda}}{m}\left(1+6 m+\mathcal{O}\left(m^{2}\right)\right) \\
w_{-1}^{(1)}+w_{-1}^{(2)} & =\left(1+\frac{15}{4} m+\mathcal{O}\left(m^{2}\right)\right) w_{-1}^{(1)} \\
& =\frac{9}{4} \frac{\hat{\lambda}}{m}\left(1+\frac{46}{9} m+\mathcal{O}\left(m^{2}\right)\right) .
\end{aligned}
$$

\section{E. Radial and angular perturbations due to a violation of the equivalence principle}

Let us relate the results (2.56) to the radial and angular perturbations of the lunar motion associated with the parameter $\hat{\lambda} \propto \bar{\delta}_{12}$. The radius vector $r=\left(X^{2}+Y^{2}\right)^{1 / 2}$ and longitude $\theta$ of the Moon (with respect to the rotating vector $\mathbf{e}_{X}=\mathbf{N}$, i.e. with respect to the Sun) are such that $u=r e^{i \theta}=\tilde{a} \zeta(1+w)$, where we recall that $\zeta=e^{i \tau}$. Hence

$$
\begin{aligned}
r^{2} & =u \bar{u}=\tilde{a}^{2}(1+w)(1+\bar{w}), \\
e^{2 i \theta} & =\frac{u}{\bar{u}}=e^{2 i \tau} \frac{1+w}{1+\bar{w}} .
\end{aligned}
$$

Working linearly in $\hat{\lambda}$ we get the following radial and longitudinal equivalence-principleviolation perturbations

$$
\begin{aligned}
& \frac{\delta_{\lambda} r}{\tilde{a}}=\Re e\left[\left(\frac{1+\bar{w}}{1+w}\right)^{1 / 2} \delta_{\lambda} w\right], \\
& \delta_{\lambda} \theta=\Im m\left[\frac{\delta_{\lambda} w}{1+w}\right] .
\end{aligned}
$$

At the approximation (2.56) we can write $\delta_{\lambda} r / \tilde{a} \simeq\left(w_{1}+w_{-1}\right) \cos \tau+\left(w_{3}+w_{-3}\right) \cos 3 \tau$, and $\delta_{\lambda} \theta \simeq\left(w_{1}-w_{-1}\right) \sin \tau+\left(w_{3}-w_{-3}\right) \sin 3 \tau$. In the approximation, the observable synodic effects are entirely described by $w_{1} \pm w_{-1}$. However, in higher approximations, $w_{ \pm 3}, w_{ \pm 5}$, etc... feed down to the synodic effects in $r$ and $\theta$. [Let us note in passing that, when averaging over time, the mean shift of the cartesian components $u=X+i Y=\tilde{a} \zeta(1+w)$ is given, to all orders, by $w_{-1}$ alone: $\langle X\rangle=\tilde{a} w_{-1},\langle Y\rangle=0$.]

Focussing on the contributions at the synodic frequency $n-n^{\prime}$, we get at this stage (in agreement with Appendix A)

$$
\begin{aligned}
\left(\frac{\delta_{\lambda} r}{\tilde{a}}\right)_{\text {synodic }} & =\frac{3}{2} \frac{\hat{\lambda}}{m}\left[1+\frac{14}{3} m+\mathcal{O}\left(m^{2}\right)\right] \cos \tau \\
\left(\delta_{\lambda} \theta\right)_{\text {synodic }} & =-3 \frac{\hat{\lambda}}{m}\left[1+\frac{16}{3} m+\mathcal{O}\left(m^{2}\right)\right] \sin \tau .
\end{aligned}
$$


A straightforward, though slightly more involved, calculation allowed us to compute by hand the $\mathcal{O}\left(\mathrm{m}^{2}\right)$ contributions to the square brackets on the right-hand sides of Eqs. (2.59). In particular, we found that the square bracket in the range perturbation, Eq. (2.59a), reads $1+\frac{14}{3} m+\frac{1061}{48} m^{2}+\mathcal{O}\left(m^{3}\right)$. In view of the large coefficients appearing in this expansion, which create large corrections to the lowest-order effect (for the Moon, $\frac{14}{3} m=0.3773$ and $\frac{1061}{48} m^{2}=0.1445$ ), we have decided to take the bull by the horns and to solve iteratively the equations of motion (2.47) to a very high order by using the dedicated computer manipulation programme MINIMS written by M. Moons from the University of Namur (Belgium) (see [46]). Some details on the application of this programme to our problem are given in Appendix B. Let us quote here the form of the results. Replacing $\hat{\lambda}$ by its definition (2.42), we see that $\tilde{a}$ drops out when writing the range perturbation $\delta_{\lambda} r$. Finally, we can write

$$
\begin{aligned}
& \left(\delta_{\lambda} r\right)_{\text {synodic }}=C(m) \bar{\delta}_{12} a^{\prime} \cos \tau, \\
& \left(\delta_{\lambda} \theta\right)_{\text {synodic }}=-C^{\prime}(m) \bar{\delta}_{12} \frac{a^{\prime}}{\tilde{a}} \sin \tau,
\end{aligned}
$$

where

$$
\begin{aligned}
& C(m)=\frac{3}{2} m\left(1+\sum_{k \geq 1} c_{k} m^{k}\right) \equiv \frac{3}{2} m S(m), \\
& C^{\prime}(m)=3 m\left(1+\sum_{k \geq 1} c_{k}^{\prime} m^{k}\right) \equiv 3 m S^{\prime}(m) .
\end{aligned}
$$

The beginning of the power series $S(m)$ entering the synodic range perturbation is

$$
\begin{aligned}
S(m)= & 1+\frac{14}{3} m+\frac{1061}{48} m^{2}+\frac{2665}{24} m^{3}+\frac{145683}{256} m^{4} \\
& +\frac{6729119}{2304} m^{5}+\frac{1656286531}{110592} m^{6}+\cdots .
\end{aligned}
$$

The coefficients of the series $S(m)$ and $S^{\prime}(m)$ are given in Appendix B up to the power $m^{17}$ included. They are found to grow fast. The ratio between two successive coefficients $c_{k} / c_{k-1}$ or $c_{k}^{\prime} / c_{k-1}^{\prime}$ is found, numerically, to converge rapidly to the value $5.1254717 \ldots$, thereby mimicking a geometric series in $m / m_{c r}$ with $m_{c r}=(5.1254717 \ldots)^{-1} \simeq 0.195103996 \ldots$. In the case of the Moon, with $m=0.0808489375 \ldots$ [47], this means that the series $S(m)$ and $S^{\prime}(m)$ converge rather slowly, as geometric series of ratio $m / m_{c r} \simeq 0.4144$. The truncation to order $m^{17}$ is just enough to estimate the values of the series to the $10^{-5}$ accuracy. We shall discuss below a method which allows us to improve this precision. We find, for the Moon,

$$
S=1.62201 \ldots, S^{\prime}=1.72348 \ldots,
$$

so that the full coefficients appearing in the synodic effects (2.60) are, respectively, $C=$ $0.196707 \ldots, C^{\prime}=0.418025 \ldots$. Finally, using the recommended value of the semi-major axis of the Earth orbit, $a^{\prime}=a_{\text {Earth }} A=1.495980221 \times 10^{13} \mathrm{~cm}$ [48] (where $A$ denotes the 
astronomical unit), the amplitude of the range oscillation of the Moon due to an equivalence principle violation is numerically found to be

$$
C a^{\prime} \bar{\delta}_{12}=2.9427 \times 10^{12} \bar{\delta}_{12} \mathrm{~cm} .
$$

In the case where one assumes the absence of violation of the weak equivalence principle, i.e. $\hat{\delta}_{A} \equiv 0$ in Eq. (2.10), the result (2.64) gives

$$
\left(\delta_{\lambda} r\right)_{\text {synodic }}=13.10 \eta \cos \tau \text { meters }
$$

if we use Eq. (2.5) 33 as nominal value for the difference of gravitational binding energies. Our final result is approximately $60 \%$ larger than the lowest-order estimate first derived by Nordtvedt in 1968 [10] and recalled in Eqs. (1.4), (1.5) and (1.8) above. On the other hand, it confirms the recent finding of Nordtvedt [25] that the interaction with the orbit's tidal deformation significantly amplifies the synodic range oscillation and substantiates it by providing explicit and accurate expressions for the amplitude of the synodic effect.

\section{PHYSICAL DISCUSSION}

\section{A. Resonances and instability}

We have seen in the previous section that the series in powers of $m$ giving the amplitudes of synodic perturbations (2.60) appear to be close to geometric series in $m / m_{c r}$ with $m_{c r} \simeq$ 0.195104. This suggests the existence of pole singularities $\propto\left(m_{c r}-m\right)^{-1}$ at $m=m_{c r} \simeq$ 0.195104 in those series. Nordtvedt [25] suggested also the presence of such a pole singularity at $m \simeq 0.2$ (i.e. for a sidereal period of about 2 months) on the basis that for such a high orbit the driving frequency ( $n^{\prime}$ in a non-rotating frame) might become equal to the rate of perigee advance $(d \varpi / d t)$. We have substantiated and generalized this suggestion, as well as obtained by several independent approaches a much more precise value for $m_{c r}$, namely,

$$
m_{c r}=0.1951039966 \ldots
$$

by making use, notably, of the work of Hénon [49] on the three-body problem. To relieve the tedium the details of our arguments are relegated to Appendix C. Let us summarize our approach and our results.

Our approach consists of putting together the (numerical) results of Hénon [49] on the stability of the periodic orbits in Hill's problem, with some knowledge of the general structure of Hamiltonian perturbations, and a more specific use of the analytical structure of the solutions of Hill's variational equations in presence of "forcing" terms, such as the ones coming from the potentials $F_{1}$, Eq. (2.29b), and $F_{3}$, Eq. (2.29d), which are neglected in Hill's main problem. Our conclusions are that when $m$ increases up to $m_{c r}$, Eq. (3.1), there is a confluence of correlated singularities: on the one hand, as found by Hénon, the free perturbations of Hill's orbit (those not driven by any additional forces) loose their stability, and on the other hand, all perturbations driven by perturbing potentials of any odd frequency in the rotating frame (i.e. containing terms $\propto \exp [ \pm(2 k+1) i \tau])$ develop pole singularities $\propto\left(m_{c r}-m\right)^{-1}$. As indicated by Nordtvedt the value $m=m_{c r}$ does correspond 
to a simple $1: 1$ commensurability $d \varpi / d t=n^{\prime}$ or $\mathrm{c} \equiv d l / d \tau=1$ in the rotating frame (where $l=n t+\epsilon-\varpi$ is the mean anomaly). Note, however, that, contrary to what happens in the familiar case of a harmonic oscillator, the basic frequency of the driving force does not need to have a $1: 1$ resonance with the natural frequency of the orbit (perigee precession) to generate poles $\propto\left(m_{c r}-m\right)^{-1}$; the odd commensurabilities $3: 1,5: 1$, etc. generate similar poles. Therefore, both the (hypothetical) equivalence-principle-violation effects (potential $F_{1}$ ), and many of the (really existing) parallactic effects (potentials $F_{3}, F_{5}, \ldots$ ) will have pole singularities $\propto\left(m_{c r}-m\right)^{-1}$. Moreover, these poles are present not only in the synodic terms (that we concentrate upon here) but in the terms at frequencies $3\left(n-n^{\prime}\right), 5\left(n-n^{\prime}\right)$, .... The consequences of this situation are explored in the following subsections.

\section{B. Padé approximant of equivalence-principle-violation effects}

The analysis of Appendix $\mathrm{C}$ shows that the amplitudes of the synodic perturbations (2.60) considered as functions of $m$ have a simple pole (but no branch point) on the positive real axis at $m=m_{c r}$, Eq. (3.1), and have no singularities on the negative real axis down to $m=-1$ (because of the stability of the retrograde orbits 49]) [0. This simple analytical behaviour suggests that the numerical validity of the power series representation (2.61) can be efficiently extended by using Padé approximants, i.e. by rewriting the power series $S(\mathrm{~m})$, $S^{\prime}(m)$ truncated at order $m^{17}$ as quotients $N(m) / D(m), N^{\prime}(m) / D^{\prime}(m)$ of two power series truncated at order $m^{8}$. The explicit coefficients of the Padé approximants 7 are given in Appendix B. We have done several checks of the conjecture that these Padé approximants provide a numerically accurate representation of the exact solution $S(m)$ on the entire interval $\left(-1, m_{c r}\right)$. First, the real zeros of smallest absolute value of the denominators $D(m)$ and $D^{\prime}(m)$ are respectively found to be $0.19510399668 \ldots$ and $0.19510399660 \ldots$ in excellent agreement with Hénon's value (3.1). Second, we found that the Padé approximants truncated to order $m^{7}$ numerically agree all over the interval $\left(-1, m_{c r}\right)$ with those at order $m^{8}$ within better than $1 \%$. [Actually, the difference is much smaller than $10^{-3}$ except very near $m=-1$.] We plot in Fig. 1 the Padé approximant of the coefficient $C(m)$ of the radial synodic effect,

$$
C_{\text {Pade }}(m)=\frac{3}{2} m \frac{N_{8}(m)}{D_{8}(m)},
$$

over the interval $\left(-1, m_{c r}\right)$. Let us note the two numerical values (using $m_{\text {Moon }}=$ $0.0808489375 \ldots ;$; 47$])$

\footnotetext{
${ }^{6}$ The value $m=-1$ corresponds to very wide retrograde orbits $0<-n \ll n^{\prime}$.

${ }^{7}$ It is to be noted that, thanks to the nearly geometric progression of the coefficients in many of the power series of Appendix B, a simpler (though less general) alternative to Padé approximants would be simply to factorize $\left(1-m / m_{c r}\right)^{-1}$.
} 


$$
\begin{aligned}
C_{\text {Pade }}\left(m_{\text {Moon }}\right) & =0.196707, \\
C_{\text {Pade }}(-1) & =-0.267706 .
\end{aligned}
$$

In Fig. 1 the lunar value (3.3a) is indicated by a dot. The difference between the linearized approximation $\frac{3}{2} m$ (dashed line in Fig.1) and the exact value of $C(m)$ (solid line) illustrates the importance of nonlinear effects in the radial synodic perturbation. 


\section{FIGURES}

FIG. 1. Coefficient $C(m)$ of the synodic range oscillation (defined in Eq. (2.60a)) as a function of $m=n^{\prime} /\left(n-n^{\prime}\right)$. The solid line represents the Padé approximant of $C(m)$, while the dashed line represents the linearized approximation $\frac{3}{2} m$. The dot indicates the actual lunar value.

\section{Better orbital tests of the equivalence principle?}

Nordtvedt [25] has suggested to make use of the resonance at $m=m_{c r}$ to improve the precision of equivalence principle tests. The idea would be to put an artificial satellite in an orbit close to the resonant orbit $\left(m=m_{c r}\right)$. From our numerical estimates, the resonant orbit has a sidereal period $T_{c r}=m_{c r}\left(1+m_{c r}\right)^{-1} T^{\prime}$ (where $T^{\prime}=2 \pi / n^{\prime}=1$ year), i.e. $T_{c r}=1.95903$ month. The corresponding "bare" semi-major axis $a_{0} \equiv\left(G m_{0} / n^{2}\right)^{1 / 3}$ is $1.68255 a_{0}$ (Moon). Though interesting, this suggestion is fraught with difficulties. First, our results show that one must be very careful to use a sub-critical orbit $\left(m<m_{c r}\right)$ as super-critical orbits are exponentially unstable (real characteristic multiplier $>1$ ). Second, the fact that all the parallactic perturbations (proportional to $m_{3}^{\text {grav }} / a^{\prime k}$ with $k \geq 4$, i.e. to $\left.m^{2}\left(\tilde{a} / a^{\prime}\right)^{k-3}\right)$ develop also pole singularities at $m=m_{c r}$ probably implies that the orbit becomes unstable slightly below the ideal Hill value (3.1) 8 . Moreover, the blow up of the parallactic perturbations amplify already large synodic effects which are known only with finite accuracy. Indeed, for some years the precision of the Lunar Laser Ranging experiment has been limited by the accuracy with which one could theoretically compute and subtract the synodic parallactic perturbations [21]. To investigate this point we have included the octupole-tide perturbation $F_{3}$, Eq. (2.29d), i.e. we added the contribution $W_{3}$, Eqs. (2.48), in our Hill-Brown iteration program. Our explicit results are given in Appendix B. The form of the radial perturbation is

$$
\begin{aligned}
\left(\delta_{\pi} r\right)_{\text {synodic }} & =\frac{15}{16} \frac{m_{2}-m_{1}}{m_{2}+m_{1}}\left(\frac{m_{1}+m_{2}}{m_{1}+m_{2}+m_{3}}\right)^{2 / 3} a^{\prime} P(m) \cos \tau \\
P(m) & =m^{7 / 3}[\kappa(m)]^{-2 / 3} Q(m) \\
Q(m) & =1+\frac{22}{5} m+\frac{215}{16} m^{2}+\frac{57599}{960} m^{3}+\frac{917401}{2880} m^{4}+\frac{230247737}{138240} m^{5}+\frac{14206254151}{1658880} m^{6}+\cdots
\end{aligned}
$$

The numerical value of the coefficient giving the scale of $\delta_{\pi} r$ in Eq. (3.4a) is $\delta_{\pi} r \simeq$ 28716.38P $(m) \cos \tau$ kilometers. The Padé approximant of the series $Q(m)$ is given in Appendix B. For rough orders of magnitude estimates we can approximate (when $0<m<m_{c r}$ ) $P(m)$ by $P(m) \simeq m^{7 / 3}\left(1-m / m_{c r}\right)^{-1}$. By comparison, the coefficient entering the Nordtvedt effect (2.60a) can be roughly approximated by $C(m) \simeq \frac{3}{2} m\left(1-m / m_{c r}\right)^{-1}$. Let us define

\footnotetext{
${ }^{8}$ Unpublished calculations of Hénon (private communication) for a small but non-zero mass ratio $\mu=m_{0} /\left(m_{0}+m_{3}\right)=10^{-6}$ show that the topology of the loss of stability of Hill's prograde orbits is different from the ideal Hill case $(\mu=0)$ and the same as for generic values of $\mu \neq 0$. The difference takes place in a region of fractional size $10^{-3}\left(\sim \mu^{1 / 2}\right.$ ?) which suggests that the actual $m_{c r}$ is roughly $0.1 \%$ smaller than the value (3.1).
} 
the amplification factor of the Nordtvedt effect as the ratio $A(m) \equiv C(m) / C\left(m_{\text {Moon }}\right)$ where $C(m)$ is the coefficient in Eq. (2.60a). The amplification factor in the synodic parallactic oscillation (3.4a) will be $B(m) \equiv P(m) / P\left(m_{\text {Moon }}\right) \simeq\left(m / m_{c r}\right)^{4 / 3} A(m)$. For an artificial satellite $\left(m_{1} \ll m_{2}\right)$ one expects from Eq. (3.4a) that the main uncertainty in the theoretical value of $\delta_{\pi} r$ will come from the Earth/Sun mass ratio: $m_{2} / m_{3}$. The current fractional uncertainty on this ratio is $\epsilon_{8} 10^{-8}$ with $\epsilon_{8} \simeq 1$ [50]. The corresponding uncertainty in $\delta_{\pi} r$ is $0.073 \epsilon_{8} B(m)$ centimeters. Therefore the use of a higher orbit, amplifying the Nordtvedt effect by a factor $A(m)$, will entail a correspondingly increased uncertainty on the synodic parallactic radial oscillation:

$$
\delta_{\pi} r \simeq 0.073 \epsilon_{8}\left(m / m_{\text {Moon }}\right)^{4 / 3} A(m) \cos \tau \mathrm{cm} .
$$

The problem might cure itself by the fact that the ratio $m_{2} / m_{3}$ will enter also the $\cos 3 \tau$ parallactic effects, which will be also amplified. But things might get complicated because, as one approaches the resonance, several frequencies become close to each other and one needs long data span to resolve the various frequencies and measure separately their Fourier coefficients. Moreover, the real motion of an artificial satellite beyond the Moon's orbit will be very complex because of the combined gravitational effects of the Earth and the Moon. Finally, such a satellite would need to be endowed with a very high performance drag free system to compete with the Moon which is, naturally, drag free to high precision.

In view of the difficulties associated with near-resonant lunar-type orbits it is worth thinking about other possibilities?. Let us list some possibilities: artificial satellites around outer planets would be interesting in that the basic dimensionful scale factor in the synodic effect (2.60a) is $a^{\prime}$, the semi-major axis of the considered planet around the Sun. That would give a factor 5 for Jupiter and a factor 10 for Saturn. In either case one would need far enough satellites (i.e. $m$ big enough) to have a coefficient $C(m)$ at least comparable to the lunar value (3.3). A second possibility is to use retrograde orbits which are always stable (in the Hill approximation). However, Fig. 1 shows that they give, at best, a factor $C(-1)=-0.267706$. An equivalence-principle mission consisting of a pair of artificial satellites around an outer planet (one prograde, one retrograde), with a laser link between the satellites, could improve by a significant factor upon the LLR experiment. Besides an improved scale factor $a^{\prime}$, the advantage of being around an outer planet is that the radiation pressure from the Sun is much smaller, so that the requirements on the drag-free system are much less stringent.

\section{Theoretical significance of orbital tests of the universality of free fall}

As we mentioned above, the Lunar Laser Ranging experiment is sensitive, through the synodic effect (2.60), to the sum of two physically independent contributions

\footnotetext{
${ }^{9}$ Let us note in passing that, because of tidal dissipation, the Moon itself is, kindly, slowly receding toward higher orbits. However, even under the overoptimistic assumption that the present rate of energy dissipation continues to apply in the future, the increase in the semi-major axis of the Moon will be only $\simeq 23 \%$ in 6 billion years (which is the expected lifetime of the Sun) 51.
} 


$$
\begin{aligned}
\bar{\delta}_{12} & =\hat{\delta}_{1}-\hat{\delta}_{2}+\eta\left(\frac{E_{1}^{\mathrm{grav}}}{m_{1} c^{2}}-\frac{E_{2}^{\mathrm{grav}}}{m_{2} c^{2}}\right) \\
& =\hat{\delta}_{12}+4.45 \eta 10^{-10} .
\end{aligned}
$$

The first contribution, $\hat{\delta}_{12} \equiv \hat{\delta}_{1}-\hat{\delta}_{2}$, is essentially equivalent to what Newton and Laplace had in mind when they proposed orbital tests of the universality of free fall: bodies of different internal compositions could fall differently. The second contribution, proportional to $\eta=4 \bar{\beta}-\bar{\gamma}$, was discovered by Nordtvedt who was assuming that the most natural theoretical framework in which to study possible macroscopic deviations from Einstein's theory is the class of metrically-coupled theories of gravity (see e.g. [19 for a review). Actually, from the perspective of modern unified theories the class of metrically-coupled theories of gravity seems quite ad hoc. For instance, string theory does suggest the possibility that there exist long-range scalar fields contributing to the interaction between macroscopic bodies and thereby modifying the standard predictions of general relativity. However, all the scalar fields present in string theory have composition-dependent couplings for very basic reasons (for a discussion of general theoretical alternatives to Einstein's theory and the types of composition-dependent couplings they might exhibit see [35]).

Recently, a mechanism has been proposed by which some of the scalar fields of string theory might survive in the macroscopic world as very weakly coupled long range fields [36] (see also [53]). In the model of Ref. [36] the surviving scalar field(s) modify the observational consequences of general relativity in several ways: (i) they violate the "weak equivalence principle" $\left(\hat{\delta}_{A} \neq 0\right)$ because of the composition-dependence of their couplings to matter; (ii) they modify the post-Newtonian $\left(\mathcal{O}\left(1 / c^{2}\right)\right)$ effects in essentially the way which is parametrized by the Eddington parameters $\bar{\nabla} \bar{\beta}$ and $\bar{\gamma}$; and (iii) they induce a slow time variability of all the coupling constants of Nature: $G, \alpha, \alpha_{\text {weak }}, \ldots$. The point we want to emphasize here, because we think it is generic, is that all those modifications of general relativity are related, because they derive ultimately from the couplings of the same field. In particular, the first term on the right-hand side of Eq. (3.6) is related to the second.

More precisely, in the model of Ref. [36], we have, for an individual atom labelled by $A$, the link

$$
\hat{\delta}_{A} \simeq-0.943 \times 10^{-5} \bar{\gamma}(E / M)_{A}
$$

where $E \equiv Z(Z-1) /(N+Z)^{1 / 3}$ is associated to the electrostatic interaction energy of the nucleus of the atom, and where $M$ denotes the mass of $A$ in atomic mass units. We believe that the structure of this link between $\hat{\delta}_{A}$ and $\bar{\gamma}(E / M)_{A} \boxplus$ is generic in string-derived models, because it follows from a basic physical feature of the massless scalar fields ("moduli") present in string theory, namely that they define the values of the gauge coupling constants.

\footnotetext{
${ }^{10}$ This comes from a feature of their couplings which is deeply rooted into the structure of QCD and the consequences it has for the mass of atoms; see pages $550-553$ of [36].

${ }^{11}$ We neglect here contributions proportional to the ratios (baryon number)/(mass) and (neutron excess)/(mass) which tend to be subdominant, even for moderate $Z$ differences 36].
} 
Even the magnitude of the numerical coefficient shoud be somewhat generic. Indeed, its analytical expression $-\frac{1}{2} a_{3} \alpha \lambda_{\alpha} / \lambda_{u_{3}}$ (in the notation of [36]) shows that it is determined by basic physical facts or assumptions: fractional smallness of electrostatic nuclear contributions $\left(a_{3} \alpha \simeq 0.770 \times 10^{-3}\right)$, unification of gauge coupling constants $\left(\lambda_{\alpha} \simeq 1\right)$ and QCD confinement $\left(\lambda_{u_{3}} \simeq \ln \left(\Lambda_{\text {string }} /(\right.\right.$ a.m.u. $\left.\left.)\right) \simeq 40.8\right)$.

We have also the model-dependent link $\bar{\beta} \simeq-10.2 \kappa \bar{\gamma}$, where the dimensionless theory parameter $\kappa$ is expected to be of order unity. $[\kappa>0$ denotes here the curvature of a coupling function around a minimum and should not be confused with the notation $\kappa(m)$ used above.] These links indicate that, in the Earth-Moon case, the gravitational binding contribution to $\bar{\delta}_{12}$ is numerically negligible compared to the composition-dependent term $\hat{\delta}_{12}$. Indeed, using Eq. (3.7) and the compositional difference between the Earth and the Moon (i.e. the difference between an Earth iron core of mass $0.32 m_{2}$ and a silica-dominated Moon [52]), we find $\hat{\delta}_{12} \simeq 0.32\left(\hat{\delta}_{\mathrm{Si}}-\hat{\delta}_{\mathrm{Fe}}\right) \simeq 2.75 \times 10^{-6} \bar{\gamma}$, while the gravitational binding energy contribution is $4.45 \eta \times 10^{-10}=-4.45(40.8 \kappa+1) \times 10^{-10} \bar{\gamma}$. From the point of view advocated here, the conclusion is that LLR data give us a very precise test of the weak equivalence principle. The loss of a Nordtvedt-type direct test of the combination $\eta=4 \bar{\beta}-\bar{\gamma}$ is compensated by the theoretically expected link $\bar{\delta}_{12} \simeq \hat{\delta}_{12} \simeq 2.75 \times 10^{-6} \bar{\gamma}$ which gives an extremely good limit on the effective Eddington parameter of the considered scalar model. More precisely, the observational limit[2] $\bar{\delta}_{12}=(-3.2 \pm 4.6) \times 10^{-13}$ recently derived from LLR data [50, [33] translates into the following observational constraint ${ }^{\square}$ on $\bar{\gamma}$ :

$$
\bar{\gamma}=(-1.2 \pm 1.7) \times 10^{-7}
$$

The recent laboratory tests of the weak equivalence principle give comparable results. Using the experimental limit $\hat{\delta}_{\mathrm{BeCu}}=(-1.9 \pm 2.5) \times 10^{-12}$ [52] and the theoretical formula (3.7) (which yields $\hat{\delta}_{\mathrm{Be} \mathrm{Cu}}=2.41 \times 10^{-5} \bar{\gamma}$ ) we find

$$
\bar{\gamma}=(-0.8 \pm 1.0) \times 10^{-7}
$$

Impressive as these limits may seem, Ref. [36] gives a motivation for pushing equivalence principle tests further because this reference estimates that a natural range for the coupling parameter $\bar{\gamma}$ is $10^{-19} \lesssim-\bar{\gamma} \lesssim 10^{-10}$. [Note, however, that if the theory parameter $\kappa$ is of order $1 / 40$ (which corresponds, in the notation of [53], to $\kappa \sim 1$ ) larger values of $-\bar{\gamma}$, of order $10^{-7}$, are expected, in agreement with [53.] In this connection, let us mention that the LLR CERGA team plans to improve the precision of the ranging down to the 2-3 millimeter level for normal points [C. Veillet, private communication]. Extracting $\bar{\delta}_{12}$ at this level will necessitate to improve the modelling of the solar radiation pressure effects which are currently believed to contribute a synodic range oscillations of approximately $0.3 \mathrm{~cm}$ [25].

\footnotetext{
${ }^{12}$ This was obtained from partial derivatives of the numerically integrated equations of motion, and therefore independently of theoretical estimates of the value of the coefficient $C(m)$ in Eq. (2.60a).
}

${ }^{13}$ We do not take into account here the theoretical constraint that $\bar{\gamma}<0$ in all scalar models. 
If this can be done, the LLR experiment will reach the level $\bar{\delta}_{12} \sim 5 \times 10^{-14}$ corresponding to the level $\bar{\gamma} \sim 10^{-8}$. It seems that significant progress in testing the equivalence principle will require space missions: either a low Earth orbiting artificial satellite dedicated to testing the weak equivalence principle, as the STEP (Satellite Test of the Equivalence Principle) mission, or, possibly, some type of orbital test such as the one suggested in Ref. 225] and the ones suggested above.

\section{ACKNOWLEDGMENTS}

We are grateful to M. Moons for providing us with the algebraic manipulator MINIMS. We thank V.I. Arnold, V.A. Brumberg, J. Chapront, B. Chauvineau, M. Hénon, J. Henrard, J. Laskar, F. Mignard, D. Ruelle, H.H. Rugh, D. Sullivan and C. Veillet for informative discussions or communications. D. V. worked on this paper while staying at the OCA/CERGA, Grasse (France) and being supported by an H. Poincaré fellowship. He is also grateful to IHES, Bures sur Yvette (France) for its kind hospitality and partial support.

\section{APPENDIX A: TRADITIONAL LUNAR PERTURBATION THEORY}

As a check on the lowest orders of the Hill-Brown calculations presented in the text, we have also investigated the mixing between equivalence-principle-violation effects and tidal effects by means of the traditional lunar perturbation theory of de Pontécoulant [24], [40]. The equations of motion corresponding to the Lagrangian $\hat{L}=\frac{1}{2} \mathbf{v}^{2}+\mu / r+R$ read

$$
\frac{d^{2} \mathbf{r}}{d t^{2}}+\mu \frac{\mathbf{r}}{r^{3}}=\frac{\partial R}{\partial \mathbf{r}}
$$

Here, $\mathbf{r} \equiv \mathbf{x}_{12} \equiv \mathbf{x}_{1}-\mathbf{x}_{2}$ is the position vector of the Moon with respect to the Earth (in an inertial, non-rotating, coordinate system), $\mu \equiv G_{12}\left(m_{1}+m_{2}\right)$ and $R=R_{1}+R_{2}+R_{3}+$ $\cdots$ is the total potential perturbing the Keplerian motion of the Moon around the Earth. [This corresponds to Eqs. (2.21), (2.22).] We consider the coplanar problem for which it is enough to solve for the radius $r \equiv|\mathbf{r}|$ and the longitude $v$ (polar angle). Decomposing the acceleration into radial and longitudinal components leads to

$$
\begin{aligned}
\ddot{r}-r \dot{v}^{2} & =-\mu r^{-2}+\partial R / \partial r \\
d\left(r^{2} \dot{v}\right) / d t & =\partial R / \partial v
\end{aligned}
$$

de Pontécoulant's method uses the longitudinal equation (A2b) but replaces the radial one (A2a) by the "virial" equation dealing with the second time derivative of $r^{2}$. The basic equations are then written as

$$
\begin{aligned}
\frac{1}{2} \frac{d^{2}}{d t^{2}}\left(r^{2}\right)-\frac{\mu}{r}+\frac{\mu}{a_{c}} & =P \\
\dot{v}-\frac{h_{c}}{r^{2}} & =\frac{1}{r^{2}} \int d t \frac{\partial R}{\partial v},
\end{aligned}
$$


where $a_{c}$ and $h_{c}$ are some integration constants and where the transformed source term in the radial equation is

$$
\begin{aligned}
P & \equiv r \frac{\partial R}{\partial r}+2 \int d t\left(\frac{d}{d t}\right)_{1} R \\
& =r \frac{\partial R}{\partial r}+2 R+2 n^{\prime} \int d t \frac{\partial R}{\partial v} .
\end{aligned}
$$

In the first form of $P,(d / d t)_{1}$ denotes a time derivative taking into account only the variability due to the time-dependence of the coordinates of the Moon: $(d / d t)_{1}=\dot{r} \partial / \partial r+\dot{v} \partial / \partial v$. The second form of $P$ is obtained by taking into account the time-dependence of $R$ upon the Sun's coordinates, and assumes that the Sun moves on a circular orbit $\left(\dot{r}^{\prime}=0, \dot{v}^{\prime}=n^{\prime}\right)$. It is very useful to notice that if $R=\sum_{p} R_{p}$ where each contribution $R_{p}\left(r, v-v^{\prime}\right)$ has a radial dependence $\propto r^{p}$, then $P=\sum_{p} P_{p}$ with

$$
P_{p}=(p+2) R_{p}+2 n^{\prime} \int d t \frac{\partial R_{p}}{\partial v} .
$$

Note that the use of the suffix $p$ is consistent with the notation $R_{1}, R_{2}, R_{3}$ of Eqs. (2.22).

First-order perturbation theory is very easy. Let us consider a general term $R_{p}^{(q)}=$ $A r^{p} \cos q\left(v-v^{\prime}\right)$, perturbing the zeroth-order (circular) solution $r=a, v_{0}=n t+\varepsilon$ (with zeroth-order integration constants $a_{c}=a, h_{c}=n a^{2}$, and the link $\left.n^{2} a^{3}=\mu\right)$. Inserting the perturbed solution $r=a+\delta r, v=v_{0}+\delta v$ into Eqs. (A3) (with perturbed integration constants $\left.a_{c}=a+\delta a, h_{c}=h+\delta h\right)$ yields

$$
\begin{aligned}
& \frac{d^{2}}{d t^{2}}(\delta r)+n^{2} \delta r=\tilde{p} A a^{p-1} \cos \left[q\left(n-n^{\prime}\right)\left(t-t_{0}\right)\right]+n^{2} \delta a, \\
& \frac{d}{d t}(\delta v)+2 \frac{n}{a} \delta r=\frac{1}{\left(n-n^{\prime}\right)} A a^{p-2} \cos \left[q\left(n-n^{\prime}\right)\left(t-t_{0}\right)\right]+a^{-2} \delta h,
\end{aligned}
$$

where

$$
\tilde{p} \equiv p+2+2 \frac{n^{\prime}}{n-n^{\prime}}=p+2+2 m
$$

Here, as in the text, we shall use $m \equiv n^{\prime} /\left(n-n^{\prime}\right)$ as the small expansion parameter of perturbation theory. The solution of Eqs. (A6) is (with $\tau \equiv v_{0}-v_{0}^{\prime}=\left(n-n^{\prime}\right)\left(t-t_{0}\right)$ )

$$
\begin{aligned}
& \delta r=\frac{\tilde{p}}{n^{2}-q^{2}\left(n-n^{\prime}\right)^{2}} A a^{p-1} \cos q \tau+\delta a, \\
& \delta v=-\frac{1}{q\left(n-n^{\prime}\right)}\left[\frac{2 \tilde{p} n}{n^{2}-q^{2}\left(n-n^{\prime}\right)^{2}}-\frac{1}{n-n^{\prime}}\right] A a^{p-2} \sin q \tau+(\delta n) t+\delta \epsilon,
\end{aligned}
$$

where $\delta n=-2 n a^{-1} \delta a+a^{-2} \delta h$. Eqs. (A6)-(A8) have been written assuming $q \neq 0$. They take a different form when $q=0$. It is traditional [40 to keep $n$ fixed throughout the approximation process (and therefore numerically equal to the observed mean motion), and to define $a$ by $n^{2} a^{3} \equiv \mu$. Then $\delta h$ is computed in terms of $\delta a$ so that $\delta n=0$. Finally, one must make use of the original radial equation (A2a) to determine $\delta a$. 
We are especially interested in the case where the perturbing potential $R$ is the sum of the quadrupole tide $R_{2}$, Eq. (2.26b), and of a term with frequency $q=1$ :

$$
R=R_{2}+R_{p}=n^{\prime 2} r^{2}\left[\frac{1}{4}+\frac{3}{4} \cos 2\left(v-v^{\prime}\right)\right]+A r^{p} \cos \left(v-v^{\prime}\right) .
$$

In the case of the equivalence-principle-violating term (2.26a the perturbing term in $\cos \left(v-v^{\prime}\right)$ has $p=1$, while the octupolar tide (2.260), for which $p=3$, contains a perturbing term in $\cos \left(v-v^{\prime}\right)$ (that we focus on) and a term in $\cos 3\left(v-v^{\prime}\right)$. At the fractional order $\mathcal{O}\left(\mathrm{m}^{2}\right)$ beyond the first-order solution (A8), the $\cos 3\left(v-v^{\prime}\right)$ term mixes with the quadrupole tides $\propto \cos 2\left(v-v^{\prime}\right)$ to generate the frequency $q=1$. As we work here only at the fractional order $\mathcal{O}(m)$ beyond the first-order solution, we do not need to study the effect of the $\cos 3\left(v-v^{\prime}\right)$ term. The first-order solution corresponding to Eq. (A9) reads

$$
\begin{aligned}
& r=a+\delta^{0} r+\delta^{2} r+\delta^{1} r, \\
& v=n t+\epsilon+\delta^{2} v+\delta^{1} v,
\end{aligned}
$$

where the superscripts on $\delta$ indicate the value of the frequency $q$. To sufficient accuracy for our purpose we have from Eqs. (A8)

$$
\begin{aligned}
\delta^{0} r+\delta^{2} r & =-m^{2} a\left(\frac{1}{6}+\cos 2 \tau\right)+\mathcal{O}\left(m^{3}\right), \\
\delta^{2} v & =\frac{11}{8} m^{2} \sin 2 \tau+\mathcal{O}\left(m^{3}\right) \\
\delta^{1} r & =C_{r}^{\text {first-order }} \frac{A}{\left(n-n^{\prime}\right)^{2}} a^{p-1} \cos \tau, \\
\delta^{1} v & =C_{v}^{\text {first-order }} \frac{A}{\left(n-n^{\prime}\right)^{2}} a^{p-2} \sin \tau
\end{aligned}
$$

with

$$
\begin{aligned}
& C_{r}^{\text {first-order }}=\frac{p+2+2 m}{2 m+m^{2}}=\frac{p+2}{2 m}\left[1+\frac{2-p}{2(2+p)} m+\mathcal{O}\left(m^{2}\right)\right], \\
& C_{v}^{\text {first-order }}=-2 \frac{(p+2+2 m)(1+m)}{2 m+m^{2}}+1=-2 \frac{p+2}{2 m}\left[1+\frac{4+p}{2(2+p)} m+\mathcal{O}\left(m^{2}\right)\right],
\end{aligned}
$$

Note that the constant term $-m^{2} a / 6$ in Eq. (A11a) depends on $\delta a=a_{c}-a$ and must be determined by having recourse to Eq. A2a . Note also that the small denominator present when $q=1, n^{2}-\left(n-n^{\prime}\right)^{2}=\left(n-n^{\prime}\right)^{2}\left[(1+m)^{2}-1\right]=\left(n-n^{\prime}\right)^{2}\left[2 m+m^{2}\right]$, causes the synodic effects to be of order $\mathcal{O}\left(\mathrm{m}^{-1} A\right)$ instead of the usual order $\mathcal{O}(A)$ valid when $q \neq 1$. One of the effects of this small denominator is to have a simple, approximate link between the radial and longitudinal synodic oscillations: $C_{v}^{\text {first-order }}=-2 C_{r}^{\text {first-order }}[1+\mathcal{O}(m)]$. Another effect is that only the leading terms in Eqs. (A12) are correct. The $\mathcal{O}(m)$ fractional corrections are modified by higher iterations as we are going to see.

When proceeding to the next iteration, several effects must be taken into account. On the left-hand side of Eq. (A3a) one must keep the terms of order $(\delta r)^{2}$, while on the righthand side one must include the change $\delta P$ of the source term $P\left(r, v-v^{\prime}\right)$ induced by the first-order solution (A11). This leads to the following equation for $\delta r=r-a$ 


$$
\begin{aligned}
a\left(\frac{d^{2}}{d t^{2}}+n^{2}\right) \delta r & =P\left(r_{0}, v_{0}-v_{0}^{\prime}\right)+\delta P_{\mathrm{eff}} \\
\delta P_{\mathrm{eff}} & =\delta P+\left(n^{2}-\frac{1}{2} \frac{d^{2}}{d t^{2}}\right)(\delta r)^{2}
\end{aligned}
$$

When computing the synodic effects with fractional accuracy $1+\mathcal{O}(m)$, the computation of $\delta P$ is simplified by several circumstances. Because of the amplification $\mathcal{O}\left(m^{-1} A\right)$ of the first-order synodic effects, one finds that it is enough to compute in Eq. (A5) the change of the first contribution, $(p+2) R_{p}$, for $p=2$ and under the synodic variations $\delta^{1} r, \delta^{1} v$. This yields

$$
\begin{aligned}
(\delta P)_{\text {synodic }} & =4\left(\delta R_{2}\right)_{\text {synodic }}=\left\{\left(\delta^{1} r \frac{\partial}{\partial r}+\delta^{1} v \frac{\partial}{\partial v}\right)\left[n^{\prime 2} r^{2}\left(1+3 \cos 2\left(v-v^{\prime}\right)\right)\right]\right\}_{\text {synodic }} \\
& =11 n^{\prime 2} a^{2} B \cos \tau[1+\mathcal{O}(m)],
\end{aligned}
$$

where $B \equiv(2 m)^{-1}(p+2)\left(n-n^{\prime}\right)^{-2} A a^{p-2}$ denotes the leading value of the fractional synodic range oscillation $\left(\delta^{1} r / a \simeq B \cos \tau, \delta^{1} v \simeq-2 B \sin \tau\right)$. We need also to extract the synodic piece of $(\delta r)^{2}=\left(\delta^{0} r+\delta^{1} r+\delta^{2} r\right)^{2}$ coming from the mixing between $\delta^{0} r+\delta^{2} r \simeq-m^{2} a\left(\frac{1}{6}+\right.$ $\cos 2 \tau)$ and $\delta^{1} r \simeq a B \cos \tau:(\delta r)_{\text {synodic }}^{2} \simeq-\frac{4}{3} a^{2} m^{2} B \cos \tau[1+\mathcal{O}(m)]$. Finally, the synodic piece of the second-order effective source term for de Pontécoulant's radial equations (A13) is obtained as

$$
\begin{aligned}
\delta^{1} P_{\text {eff }} & =(\delta P)_{\text {synodic }}+\left[n^{2}+\frac{1}{2}\left(n-n^{\prime}\right)^{2}\right]\left(\delta r^{2}\right)_{\text {synodic }} \\
& =9 n^{\prime 2} a^{2} B \cos \tau[1+\mathcal{O}(m)] .
\end{aligned}
$$

The corresponding solution reads

$$
\begin{aligned}
\left(\frac{\delta^{1} r}{a}\right)^{\text {second-order }} & \simeq 9 \frac{n^{2}}{n^{2}-\left(n-n^{\prime}\right)^{2}} B \cos \tau \\
& \simeq \frac{9}{2} m B \cos \tau
\end{aligned}
$$

When turning to the longitude equation (A3b) one finds also some simplifications: the change of the source term $\propto \int d t \partial R / \partial v$ is of order $m^{2} \times m^{-1} A=\mathcal{O}(m A)$. The corresponding term in the solution is not amplified by a small denominator and is therefore negligible compared to the precision $m \times m^{-1} A=\mathcal{O}(A)$ we are aiming for. It is therefore sufficient to integrate the equation $d / d t\left(\delta^{1} v\right)^{\text {second-order }} \simeq-2 n a^{-1}\left(\delta^{1} r\right)^{\text {second-order }}$. The final result can be very simply expressed in saying that the second iteration leads to multiplying the first-order synodic perturbations (A11d), (A11d) by the common factor $1+\frac{9}{2} m+\mathcal{O}\left(m^{2}\right)$.

In conclusion, the mixing between the quadrupole tide $R_{2}$ and some synodic-frequency perturbation potential (in which we factorize an effective gravitational mass of the Sun, $\left.G m^{\prime} \equiv n^{\prime 2} a^{\prime 3}\right)$

$$
\left(R_{p}\right)_{\text {synodic }}=\beta \frac{G m^{\prime}}{a^{\prime}}\left(\frac{r}{a^{\prime}}\right)^{p} \cos \left(v-v^{\prime}\right)
$$


leads, when neglecting non-linearities in the dimensionless parameter $\beta$ (which should not be confused with its post-Newtonian homonym), to the following synodic oscillations

$$
\begin{aligned}
\frac{\delta^{1} r}{a} & =C_{r} \beta m^{2}\left(\frac{a}{a^{\prime}}\right)^{p-2} \cos \tau \\
\delta^{1} v & =C_{v} \beta m^{2}\left(\frac{a}{a^{\prime}}\right)^{p-2} \sin \tau
\end{aligned}
$$

where

$$
\begin{aligned}
C_{r} & =\left[1+\frac{9}{2} m+\mathcal{O}\left(m^{2}\right)\right] C_{r}^{\text {first-order }} \\
& =\frac{2+p}{2 m}\left[1+\frac{10+4 p}{2+p} m+\mathcal{O}\left(m^{2}\right)\right], \\
C_{v} & =\left[1+\frac{9}{2} m+\mathcal{O}\left(m^{2}\right)\right] C_{v}^{\text {first-order }} \\
& =-2 \frac{2+p}{2 m}\left[1+\frac{11+5 p}{2+p} m+\mathcal{O}\left(m^{2}\right)\right] .
\end{aligned}
$$

The two cases of interest are: (i) an hypothetical violation of the equivalence principle in which (comparing Eq. (A17) with Eq. (2.26a))

$$
p=1, \quad \beta_{\mathrm{EP}}=\bar{\delta}_{12},
$$

and, (ii) the octupolar tide ("parallactic effects"), Eq. 2.260), with

$$
\begin{aligned}
R_{3} \simeq & \frac{1}{8}\left(X_{2}-X_{1}\right) \frac{G m^{\prime}}{a^{\prime}}\left(\frac{r}{a^{\prime}}\right)^{3} \\
& \times\left[3 \cos \left(v-v^{\prime}\right)+5 \cos 3\left(v-v^{\prime}\right)\right],
\end{aligned}
$$

whose synodic piece has

$$
p=3, \quad \beta_{\mathrm{PAR}}=\frac{3}{8}\left(X_{2}-X_{1}\right)
$$

The results (A18)-(A22) agree with the (much more accurate) Hill-Brown-type results given in the text and in Appendix B.

\section{APPENDIX B: HILL'S EQUATIONS - MORE ON THE ITERATIVE SCHEME OF SOLUTION AND NUMERICAL RESULTS}

In this Appendix, we explain in detail the iterative scheme we employed for solving Hill's equations (2.47) with the source terms (2.46), and also with the parallactic perturbation (2.48). We also give tables of the obtained solution for several physically interesting quantities. Obviously, one can envisage several iterative methods for solving the considered equations. We do not claim that the scheme we adopted is the optimal one, but we found it suitable from the point of view of memory and computing time requests. Thanks to our 
use of the dedicated algebraic manipulator MINIMS we could obtain the solution to a very high order in the formally small parameters. In what follows, we shall present the solution for the perturbation of Hill's variational orbit related to the equivalence principle violation. Exactly the same scheme applies in the case of the parallactic perturbations.

Keeping the notation of Sec. II.D, notably $L(w, \bar{w})$ for the linear operator (2.46), we have to solve

$$
\begin{aligned}
L(w, \bar{w})= & -\hat{\lambda} \zeta^{-1}-\frac{3}{2} m^{2} \zeta^{-2} \\
& -\frac{3}{2} m^{2} \bar{w} \zeta^{-2}+\kappa(m) Q(w, \bar{w}),
\end{aligned}
$$

where

$$
Q(w, \bar{w})=(1+w)^{-1 / 2}(1+\bar{w})^{-3 / 2}-1+\frac{1}{2} w+\frac{3}{2} \bar{w} .
$$

The nonlinear function $Q$ can be written as

$$
\begin{aligned}
Q(w, \bar{w})= & \sum_{k \geq 2} \chi_{k}\left(w^{k}+(2 k+1) \bar{w}^{k}\right) \\
& +\sum_{j, k \geq 1}(2 k+1) \chi_{j} \chi_{k} w^{j} \bar{w}^{k} .
\end{aligned}
$$

Here, $\chi_{k} \equiv\left(\begin{array}{c}-1 / 2 \\ k\end{array}\right)$ and $(2 k+1) \chi_{k}=\left(\begin{array}{c}-3 / 2 \\ k\end{array}\right)$ are binomial coefficients.

As in Sec. II.D we look for a formal power series solution of Eq. (B1),

$$
w=w^{(1)}+w^{(2)}+w^{(3)}+\cdots,
$$

and similarly for the complex conjugate. The superscripts on the consecutive terms in (B4) refer to corresponding orders in the combined formal small parameter $\left(\hat{\lambda}+m^{2}\right)$. Keeping track of the orders in this formal small parameter, we decompose the nonlinear source $Q$ as

$$
Q(w, \bar{w})=Q^{(2)}+Q^{(3)}+\cdots
$$

where the individual terms include symbolically

$$
Q^{(i)}=\sum_{j a+k b=i}(\text { coefficient })\left(w^{(a)}\right)^{j}\left(\bar{w}^{(b)}\right)^{k}
$$

For any particular value of $i$ in $(\mathbb{B 6}), Q^{(i)}$ is given by a finite number of terms which depend only on the knowledge of $w^{(a)}$ for $a<i$. Although the procedure of breaking $Q(w, \bar{w})$ into a sum of equal-order terms $Q^{(i)}$ might seem laborious, it is relatively easy to be programmed using a well suited algebraic manipulator such as MINIMS. One can introduce a formal index which conserves the order of a particular term and manipulate it like any other variable.

The heart of our iteration scheme consists of the following infinite system of differential equations 


$$
\begin{aligned}
L\left(w^{(1)}, \bar{w}^{(1)}\right) & =-\hat{\lambda} \zeta^{-1}-\frac{3}{2} m^{2} \zeta^{-2} \\
L\left(w^{(2)}, \bar{w}^{(2)}\right) & =-\frac{3}{2} m^{2} \bar{w}^{(1)} \zeta^{-2}+\kappa Q^{(2)}\left(w^{(1)}\right) \\
L\left(w^{(3)}, \bar{w}^{(3)}\right) & =-\frac{3}{2} m^{2} \bar{w}^{(2)} \zeta^{-2}+\kappa Q^{(3)}\left(w^{(1)}, w^{(2)}\right) \\
\ldots & =\ldots \\
L\left(w^{(k)}, \bar{w}^{(k)}\right) & =-\frac{3}{2} m^{2} \bar{w}^{(k-1)} \zeta^{-2}+\kappa Q^{(k)}\left(w^{(1)}, w^{(2)}, \ldots, w^{(k-1)}\right) \\
\ldots & =\ldots .
\end{aligned}
$$

It is easy to verify, that the generic form of the terms in the right-hand sides of Eqs. (B7) reads $W_{k} \zeta^{k}+W_{-k} \zeta^{-k}$ as presented in (2.50). The unique inversion of the linear operator $L$ on the left-hand sides of Eqs. (B7) is given by formulas (2.51)-(2.52). Notice also that suppressing the equivalence-principle-violation term $(\hat{\lambda}=0$ in $(\sqrt[B]{B}))$, one recovers a system of equations for constructing the usual variational periodic orbit.

In the preceding scheme, we consider $\hat{\lambda}$ and $m^{2}$ as two comparable "small" parameters. However, in practice, the order of the $\hat{\lambda}$ parameter associated with the studied violation of the equivalence principle is numerically much smaller than $m^{2}$ (which can be as large as 1). As a result, we restrict the generality of our solution by keeping only the first order in the parameter $\hat{\lambda}$. This truncation allows a clear separation in the interpretation of the oddand even-power terms in the $\zeta$ variable of the final solution for $w$ (and $\bar{w}$ ): (i) the evenpower terms $\left(\propto \zeta^{2 i}\right)$ never contain the perturbation parameter $\hat{\lambda}$ and fully reconstruct Hill's variational solution, (ii) the odd-power terms $\left(\propto \zeta^{2 i+1}\right)$ are all of the first-order in $\hat{\lambda}$ (but they are coupled to the "background" variational terms through an infinite series of powers of $m^{2}$ ). We thus simultaneously obtain Hill's variational solution and its $\hat{\lambda}$-perturbation by filtering the various powers of $\zeta$. This is a particularly important circumstance, because the series giving the variational solution enters the definition of several studied quantities such as the radial or longitudinal perturbations of the lunar orbit by the equivalence-principleviolation terms (see Eqs. (2.58)).

Once the iterative scheme is set up and the numerical program debugged, we can obtain the solution of our problem up to an arbitrary order. The limits of the solution are then given mainly by the computer power. A minor limit comes from the fact that the MINIMS algebraic manipulator works with double precision real coefficients (16 digits) [46]. During the manipulation of the series, one thereby accumulates round-off errors. However, we have checked that this restriction is not significant for our work ${ }^{\text {th }}$.

In the rest of this Appendix we shall present tables of the numerical coefficients achieved by the previous algorithm for different series introduced in the main text of the paper and related to physical quantities.

Let us start with our solution for the variational curve $(\hat{\lambda}=0)$. Tables I and II give the

14 We have performed a lower order solution in using the modified version of the distributed MINIMS manipulator which accepts the quadruple precision (32 digits) for the coefficients of the series and compared it with the double precision one. 
coefficients $w_{i j}$ of the double series expansion of $w: w=\sum w_{j k} \zeta^{j} m^{k}$ where $j=0, \pm 2, \pm 4, \ldots$; $k=2,3,4, \ldots$ and $|j| \leq k$ ( $j$ labels the rows and $k$ the columns). Contrary to the method of Ref. [17] the $\zeta$-independent term is not fixed to unity. However, due to the choice of the fiducial semimajor axis $\tilde{a}$, defined in Eq. (2.36), it starts only at the power $m^{4}$. 


\section{TABLES}

TABLE I. Coefficients $w_{j k}$ of the double series giving Hill's variational curve: $w=\sum w_{j k} \zeta^{j} m^{k}$; $j$ labels the rows and $k$ the columns.

\begin{tabular}{|c|c|c|c|c|c|}
\hline & 2 & 3 & 4 & 5 & 6 \\
\hline$\overline{-6}$ & - & - & - & - & 0.005208333333333333 \\
\hline-4 & - & - & 0.0000000000000000 & 0.03593750000000000 & 0.1245833333333333 \\
\hline-2 & -1.187500000000000 & -1.6666666666666667 & -1.194444444444444 & -0.5185185185185185 & -0.8265365788966050 \\
\hline 0 & - & - & 0.6210937500000000 & -0.6770833333333333 & -0.6892361111111111 \\
\hline 2 & 0.1875000000000000 & 0.5000000000000000 & 0.5833333333333333 & 0.3055555555555556 & -0.1615849247685185 \\
\hline 4 & - & - & 0.0976562500000000 & 0.4182291666666667 & 0.8484722222222222 \\
\hline 6 & - & - & - & - & 0.06778971354166667 \\
\hline
\end{tabular}

TABLE II. Continuation of Table I.

\begin{tabular}{rccccc}
\hline \hline & 7 & 8 & 9 & 10 & 11 \\
\hline-10 & - & - & - & 0.002728271484375000 & 0.02314830588154192 \\
-8 & - & 0.003743489583333333 & 0.02753602458235899 & 0.09640929637552359 & 0.2138120808825399 \\
-6 & 0.03477027529761905 & 0.1040162627551020 & 0.1880280591858106 & 0.2403927412241086 & 0.2416131234195372 \\
-4 & 0.1956215277777778 & 0.2153863326461227 & 0.2955465183221726 & 0.3769043026659738 & -0.1199168976723571 \\
-2 & 1.205252137988683 & 7.122740269204389 & 15.70605739258259 & 19.78110897697562 & 14.66018287215882 \\
0 & -1.605902777777778 & -0.5700574333285108 & 0.3975170245386445 & 1.670582431118184 & 7.457045029767965 \\
2 & -1.034723427854938 & -3.070661490483539 & -6.516200630679869 & -9.530344734021654 & -7.910677339637028 \\
4 & 1.038887731481481 & 0.7041097601996523 & -0.5710030937052194 & -4.063905605879704 & -11.57027457856626 \\
6 & 0.3898297991071429 & 1.087330552012472 & 1.926893073875549 & 2.301395728392179 & 1.149297956477343 \\
8 & - & 0.05397033691406250 & 0.3869401996638499 & 1.360005033209030 & 3.095895579274965 \\
10 & - & - & - & 0.04656556447347005 & 0.3988932608444074 \\
\hline \hline
\end{tabular}


We then give the lunar orbit perturbations due to a hypothetical equivalence principle violation (terms linear in $\hat{\lambda}$ ).

Tab. III gives the coefficients $c_{k}$ of the series in powers of $m$ giving $w_{-1}$, i.e. the coefficient of $\zeta^{-1}$ in the Laurent expansion of $\delta_{\lambda} w(\zeta)$, after factorization of the leading term $\frac{9}{4}(\hat{\lambda} / m)$ (see Eq. (2.56b)). The second column $-p_{k}$ - gives the numerical value of $c_{k} m^{k}$ (in \%) for the lunar orbit $\left(m=m_{\text {Moon }}=0.0808489375 \ldots\right)$. The last column $-r_{k}$ - gives the ratio $c_{k-1} / c_{k}$ of the successive coefficients of the series (the same structure is conserved also for

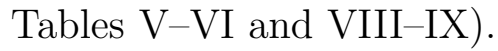

TABLE III. Coefficients of $m^{k}$ in the lunar orbit perturbation $w_{-1}$. The mark $<$ signifies that the value is smaller than $0.001 \%$.

\begin{tabular}{rccc}
\hline \hline$k$ & $c_{k}$ & $p_{k}$ & $r_{k}$ \\
\hline 0 & 1.000000000000000 & - & - \\
1 & 5.111111111111111 & 41.323 & 0.1956521739 \\
2 & 25.52777777777778 & 16.686 & 0.2002176279 \\
3 & 129.5277777777778 & 6.845 & 0.1970834227 \\
4 & 663.1076388888889 & 2.833 & 0.1953344679 \\
5 & 3400.509837962963 & 1.175 & 0.1950024174 \\
6 & 17434.56978202160 & 0.487 & 0.1950440923 \\
7 & 89366.97811374742 & 0.202 & 0.1950896198 \\
8 & 458049.7173103071 & 0.084 & 0.1951032273 \\
9 & 2347711.432406117 & 0.035 & 0.1951047778 \\
10 & 12033105.92287766 & 0.014 & 0.1951043602 \\
11 & 61675313.45886662 & 0.006 & 0.1951040902 \\
12 & 316115045.4115870 & 0.002 & 0.1951040115 \\
13 & 1620238694.871019 & 0.001 & 0.1951039970 \\
14 & 8304487501.579596 & $<$ & 0.1951039958 \\
15 & 42564415192.16274 & $<$ & 0.1951039962 \\
16 & 218162702733.8958 & $<$ & 0.1951039965 \\
17 & 1118186744062.939 & $<$ & 0.1951039966 \\
\hline \hline
\end{tabular}


Tab. IV gives the coefficients $c_{k}$ of the series in powers of $m$ giving $w_{1}$, i.e. the coefficient of $\zeta$ in the Laurent expansion of $\delta_{\lambda} w$, after factorization of the leading term $-\frac{3}{4}(\hat{\lambda} / m)$ (see Eq. (2.56a)).

TABLE IV. Coefficients of $m^{k}$ in the lunar orbit perturbation $w_{1}$. Notation as in Table III.

\begin{tabular}{rccc}
\hline \hline$k$ & $c_{k}$ & $p_{k}$ & $r_{k}$ \\
\hline 0 & 1.000000000000000 & - & - \\
1 & 6.000000000000000 & 48.509 & 0.1666666667 \\
2 & 29.62500000000000 & 19.365 & 0.2025316456 \\
3 & 147.5000000000000 & 7.795 & 0.2008474576 \\
4 & 749.9427083333333 & 3.204 & 0.1966816910 \\
5 & 3843.245659722222 & 1.328 & 0.1951326495 \\
6 & 19711.47459129051 & 0.551 & 0.1949750457 \\
7 & 101057.1821729118 & 0.228 & 0.1950526837 \\
8 & 517989.9317152261 & 0.095 & 0.1950948773 \\
9 & 2654938.425605692 & 0.039 & 0.1951043108 \\
10 & 13607759.81112155 & 0.016 & 0.1951047389 \\
11 & 69746091.35545381 & 0.007 & 0.1951042639 \\
12 & 357481499.9590677 & 0.003 & 0.1951040581 \\
13 & 1832261194.517921 & 0.001 & 0.1951040065 \\
14 & 9391202741.857013 & $<$ & 0.1951039973 \\
15 & 48134343317.40093 & $<$ & 0.1951039963 \\
16 & 246711211454.4727 & $<$ & 0.1951039964 \\
17 & 1264511316090.325 & $<$ & 0.1951039966 \\
\hline \hline
\end{tabular}


Some general properties of the presented results, already commented upon in the main text of the paper and in Appendix C, are: (i) a fast ("geometric-like") growth of the coefficients $c_{k}$ of the series with a surprisingly rapid approach of the $r_{k}\left(=c_{k-1} / c_{k}\right)$ ratio to the $m_{c r}$ value of about $0.1951039966 \ldots$. (ii) a very substantial contribution of the non-linear terms $(k \geq 1)$ in the series for the lunar value of Hill's parameter $m_{\mathrm{Moon}}$.

TABLE V. Coefficients of the $S(m)$ series yielding the radial perturbation, with synodic ( $\tau$ ") period, of the variational curve due to an equivalence principle violation.

\begin{tabular}{rccc}
\hline \hline$k$ & $c_{k}$ & $p_{k}$ & $r_{k}$ \\
\hline 0 & 1.000000000000000 & - & - \\
1 & 4.666666666666667 & 37.730 & 0.21428571429 \\
2 & 22.10416666666667 & 14.449 & 0.21112158341 \\
3 & 111.0416666666667 & 5.868 & 0.19906191370 \\
4 & 569.0742187500000 & 2.431 & 0.19512686221 \\
5 & 2920.624565972222 & 1.001 & 0.19484675483 \\
6 & 14976.54921694155 & 0.418 & 0.19501318519 \\
7 & 76767.66017493731 & 0.173 & 0.19508930170 \\
8 & 393469.7706768071 & 0.072 & 0.19510434065 \\
9 & 2016707.919972300 & 0.030 & 0.19510498609 \\
10 & 10336561.13767503 & 0.012 & 0.19510433819 \\
11 & 52979731.33709500 & 0.005 & 0.19510406861 \\
12 & 271546096.6185635 & 0.002 & 0.19510400627 \\
13 & 1391801807.779853 & 0.001 & 0.19510399764 \\
14 & 7133640668.991231 & $<$ & 0.19510399701 \\
15 & 36563272849.28028 & $<$ & 0.19510399680 \\
16 & 187404017729.6833 & $<$ & 0.19510399666 \\
17 & 960533976540.2227 & $<$ & 0.19510399664 \\
\hline \hline
\end{tabular}


TABLE VI. Coefficients of the $S^{\prime}(m)$ series yielding the longitudinal perturbation, with synodic (" $\tau$ ") period, of the variational curve due to an equivalence principle violation.

\begin{tabular}{rccc}
\hline \hline$k$ & $c_{k}$ & $p_{k}$ & $r_{k}$ \\
\hline 0 & 1.000000000000000 & - & - \\
1 & 5.333333333333333 & 43.119 & 0.18750000000 \\
2 & 26.39583333333333 & 17.254 & 0.20205209155 \\
3 & 132.9166666666667 & 7.024 & 0.19858934169 \\
4 & 678.3172743055553 & 2.898 & 0.19595058493 \\
5 & 3478.588686342593 & 1.202 & 0.19499783834 \\
6 & 17838.85192117573 & 0.498 & 0.19500070418 \\
7 & 91447.83453394253 & 0.206 & 0.19507134326 \\
8 & 468721.9555645895 & 0.086 & 0.19510038616 \\
9 & 2402405.984973695 & 0.035 & 0.19510522305 \\
10 & 12313419.50072995 & 0.015 & 0.19510469735 \\
11 & 63112018.54675127 & 0.006 & 0.19510419385 \\
12 & 323478806.4318246 & 0.003 & 0.19510402936 \\
13 & 1657981435.159388 & 0.001 & 0.19510399789 \\
14 & 8497936880.202027 & $<$ & 0.19510399507 \\
15 & 43555934597.05303 & $<$ & 0.19510399579 \\
16 & 223244707500.3778 & $<$ & 0.19510399635 \\
17 & 1144234415604.679 & $<$ & 0.19510399657 \\
\hline \hline
\end{tabular}


Tables $\mathrm{V} \nabla \mathbf{V 1}$ give the coefficients of the power series $S(m)$ and $S^{\prime}(m)$ related, respectively, to the radial and longitudinal perturbations (with synodic periodicity) of the lunar orbit due to a hypothetical violation of the equivalence principle. The coefficients of the Padé approximants of those series are given in Tab. VII. More precisely, we denote $N_{8}(m) \equiv \sum_{0}^{8} a_{k} m^{k}$ and $D_{8}(m) \equiv \sum_{0}^{8} b_{k} m^{k}$ the Padé approximants for the $S(m)$ series (see Eq. (3.2)), and $N_{8}^{\prime}(m) \equiv \sum_{0}^{8} a_{k}^{\prime} m^{k}$ and $D_{8}^{\prime}(m) \equiv \sum_{0}^{8} b_{k}^{\prime} m^{k}$ the Padé approximants for the $S^{\prime}(m)$ series. The denominator polynomials $D_{8}(m)$ and $D_{8}^{\prime}(m)$ have $0.1951039967 \ldots$ and $0.1951039966 \ldots$, respectively, as real roots.

TABLE VII. Coefficients of the Padé approximants of order 8 of the radial and longitudinal perturbation series, with synodic (" $\tau$ ") period, of the variational curve due to an equivalence principle violation.

\begin{tabular}{ccccc}
\hline \hline$k$ & $a_{k}$ & $b_{k}$ & $a_{k}^{\prime}$ & $b_{k}^{\prime}$ \\
\hline 0 & 1.000000000000000 & 1.000000000000000 & 1.000000000000000 & 1.000000000000000 \\
1 & -4.679659654425508 & -9.346326321092175 & -2.948114664716526 & -8.281447998049859 \\
2 & 9.706375765630793 & 31.21839859739427 & 2.472472675594219 & 20.24436199852680 \\
3 & -12.09649460259615 & -62.231266666796116 & -0.035120215085467699 & -22.32599642537073 \\
4 & 9.264485113438459 & 78.37781038863183 & -0.8074761600241968 & 6.322888291078106 \\
5 & -0.8206525542778040 & -59.42770492795888 & 1.129701196880901 & 4.768342316076093 \\
6 & -4.562346877625465 & 5.529574170471975 & 1.916287015273362 & 13.88318783117331 \\
7 & 0.6450458113816286 & 30.28114298289518 & -3.678767273115525 & -38.01361281338056 \\
8 & 0.9269455883562498 & -30.33613801897244 & -3.906968891383218 & 34.97077854522613 \\
\hline \hline
\end{tabular}


Tab. VIII gives the coefficients of the $S^{\prime \prime}(m)$ series yielding the equivalence-principleviolation perturbation of the lunar orbit with one-third of the synodic period (" $3 \tau$ "). This series is defined by

$$
\left(\delta_{\lambda} r\right)_{\text {third-synodic }}=C^{\prime \prime}(m) \bar{\delta}_{12} a^{\prime} \cos 3 \tau,
$$

with

$$
C^{\prime \prime}(m)=\frac{51}{32} m^{3}\left(1+\sum_{k \geq 1} c_{k}^{\prime \prime} m^{k}\right) \equiv \frac{51}{32} m^{3} S^{\prime \prime}(m) .
$$

Note that the third-synodic effect (B8) is $\mathcal{O}\left(m^{2}\right)$ smaller than the synodic effect (2.60). For instance, if we assume $\hat{\delta}_{12}=0$ the result (B) gives numerically $\left(\delta_{\lambda} r\right)_{\text {third-synodic }} \simeq$ $6.62 \eta \cos 3 \tau$ centimeters for the lunar orbit. Although there is probably no practical use of this higher frequency excitation (and of the others with frequencies $5 \tau, 7 \tau, \ldots$ ), two points are to be mentioned: (i) a significantly smaller amplitude of the effect, (ii) the persistence of the pole singularity at $m=m_{c r}$ for these odd-multiples of the basic synodic frequency as discussed in Appendix C.

TABLE VIII. Coefficients of the $S^{\prime \prime}(m)$ series yielding the radial perturbation, with third-synodic (" $3 \tau$ ") period, of the variational curve due to an equivalence principle violation.

\begin{tabular}{rccc}
\hline \hline$k$ & $c_{k}^{\prime \prime}$ & $p_{k}$ & $r_{k}$ \\
\hline 1 & 1.000000000000000 & - & - \\
2 & 6.450980392156863 & 52.155 & 0.15501519757 \\
3 & 32.60457516339869 & 21.312 & 0.19785506665 \\
4 & 163.0646514161220 & 8.618 & 0.19994876192 \\
5 & 828.6388478122731 & 3.540 & 0.19678615340 \\
6 & 4240.758469846889 & 1.465 & 0.19539873674 \\
7 & 21736.94075865620 & 0.607 & 0.19509454053 \\
8 & 111428.4766430605 & 0.252 & 0.19507527531 \\
9 & 571159.5105168029 & 0.104 & 0.19509169434 \\
10 & 2927510.871437882 & 0.043 & 0.19510073083 \\
11 & 15004916.03283528 & 0.018 & 0.19510344910 \\
12 & 76907280.99332377 & 0.007 & 0.19510397246 \\
13 & 394186033.1895487 & 0.003 & 0.19510402327 \\
14 & 2020389145.708269 & 0.001 & 0.19510401450 \\
15 & 10355446785.91400 & $<$ & 0.19510400541 \\
16 & 53076547791.36041 & $<$ & 0.19510400011 \\
17 & 272042338525.2278 & $<$ & 0.19510399771 \\
\hline \hline
\end{tabular}


Tab. [X] lists the coefficients of the $Q(m)$ series giving the radial parallactic inequality of the lunar motion $\left(Q(m) \equiv 1+\sum_{k \geq 1} q_{k} m^{k}\right)$ as defined in Eqs. (3.4) of the text. Similarly to the treatment of the equivalence-principle-violation lunar perturbation we improved on our solution by using Padé approximants. Tab. \ yields the coefficients of the corresponding polynomials. We also computed the corresponding lunar parallactic inequality in longitude. As a partial check on our results we have compared the latter with the result by Deprit, Henrard and Rom [55]. When substituting the current recommended values of the mass constants and the Earth semi-major axis we obtain 125." 438 for the amplitude of the parallactic inequality in longitude when truncating our series to the power $m^{7}$. This value is to be compared with 125." 4201 reported in Ref. [55]. We believe that the origin of the minor discrepancy between those results lays in the slightly different values of the astronomical constants employed by Deprit, Henrard and Rom at the end of the sixties.

TABLE IX. Radial perturbation of the variational curve due to parallactic terms with synodic (" $\tau$ ") period.

\begin{tabular}{rccc}
\hline \hline$k$ & $q_{k}$ & $p_{k}$ & $r_{k}$ \\
\hline 0 & 1.000000000000000 & - & - \\
1 & 4.400000000000000 & 35.574 & 0.2272727273 \\
2 & 13.43750000000000 & 8.783 & 0.3274418605 \\
3 & 59.99895833333333 & 3.171 & 0.2239622216 \\
4 & 318.5420138888889 & 1.361 & 0.1883549288 \\
5 & 1665.565227141204 & 0.575 & 0.1912515996 \\
6 & 8563.762388478974 & 0.239 & 0.1944898926 \\
7 & 43904.44785527987 & 0.099 & 0.1950545516 \\
8 & 225048.4315368017 & 0.041 & 0.1950888862 \\
9 & 1153503.664577334 & 0.017 & 0.1950998843 \\
10 & 5912167.013677382 & 0.007 & 0.1951067454 \\
11 & 30302311.46598434 & 0.003 & 0.1951061397 \\
12 & 155313062.3650712 & 0.001 & 0.1951047195 \\
\hline \hline
\end{tabular}

TABLE X. Coefficients of the Padé approximants of order 6 of the radial and longitudinal perturbations of the variational curve due to the parallactic terms with synodic (" $\tau$ ") period.

\begin{tabular}{ccccc}
\hline \hline$k$ & $a_{k}^{\prime \prime}$ & $b_{k}^{\prime \prime}$ & $a_{k}^{\prime \prime \prime}$ & $b_{k}^{\prime \prime \prime}$ \\
\hline 0 & 1.000000000000000 & 1.000000000000000 & 1.000000000000000 & 1.000000000000000 \\
1 & -2.698270836202914 & -7.098270836202914 & -1.839002476253125 & -7.039002476253125 \\
2 & -6.296177502041919 & 11.49871417725090 & -7.776514253041273 & 11.36379862347498 \\
3 & 9.635571285915953 & -5.574715065844693 & 4.712486681328506 & -6.420060419171178 \\
4 & 11.54422294537151 & -11.09416027055608 & 8.382766821277098 & -10.90642205141127 \\
5 & -7.427911939834412 & 21.91801429175468 & -4.643821407637831 & 18.97128636175652 \\
6 & -10.77769175983123 & -27.61496107523247 & -3.937875173153431 & -20.15607339834716 \\
\hline \hline
\end{tabular}




\section{APPENDIX C: CHARACTERISTIC MULTIPLIERS, COMMENSURABILITIES AND INSTABILITY}

Let us first recall the basic concept of characteristic multipliers. The small perturbations around a periodic orbit in the restricted three-body problem can be described in terms of a two-dimensional Poincaré map: this is the application connecting two successive intersections of the trajectory in phase space with a two-plane transversal to the orbit. [One works, say, in the three-dimensional reduced phase space corresponding to a fixed value of the Jacobi integral; see [54 for a catalog of such Poincaré maps in the case of the Hill problem.] For infinitesimal perturbations, the Poincaré map reduces to a linear transformation of the plane, leaving fixed the origin which corresponds to the reference periodic orbit. The two eigenvalues $\left(\lambda_{1}, \lambda_{2}\right)$ of the infinitesimal Poincaré map (a $2 \times 2$ matrix) are the characteristic multipliers. From the Hamiltonian nature of the dynamics it follows that these multipliers are either of the form $\left(e^{i \alpha}, e^{-i \alpha}\right)$ or $\left(\varepsilon e^{\beta}, \varepsilon e^{-\beta}\right)$ with $\varepsilon= \pm 1$ [57]. The first case means generally (apart from the exceptional cases where $\alpha=2 \pi / 3$, or where $\alpha=2 \pi / 4$ and some inequality is not satisfied) that the periodic orbit is (quasi-)stable. The second case means that the periodic orbit is unstable. A useful quantity for studying the possible loss of stability is half the sum of the multipliers: $a \equiv \frac{1}{2}\left(\lambda_{1}+\lambda_{2}\right)$, which is either $\cos \alpha$ (in which case $|a| \leq 1$ ) or $\varepsilon \cosh \beta$ (in which case $|a| \geq 1$ ). The loss of stability can occur (apart from the above mentioned exceptional cases) only when $a$ crosses the values \pm 1 . The stability of all the periodic orbits in Hill's problem has been studied by Hénon 449. We are interested here in the families " $g$ " and " $f$ ' of periodic orbits which correspond, respectively, to prograde and retrograde lunar-type orbits. Hénon found that retrograde orbits are stable for all values of the Jacobi integral (i.e. $-1<m<0$ ), while prograde orbits are stable only for close enough orbits, $0<m<m_{c r}$ with

$$
m_{c r}=0.1951039966 \ldots
$$

For this value $a$ crosses the value +1 .

Let us translate this result in terms of the perigee precession of a perturbed Hill orbit. Perturbations of Hill's orbit can be described in terms of the (isoenergetic) normal displacement $q=(\dot{X} \delta Y-\dot{Y} \delta X) /\left(\dot{X}^{2}+\dot{Y}^{2}\right)^{1 / 2}$. This variable satisfies "Hill's equation"

$$
\frac{d^{2} q(\tau)}{d \tau^{2}}+\Theta(\tau) q(\tau)=\sigma(\tau)
$$

where $\Theta(\tau)=\theta_{0}+2 \sum_{1}^{\infty} \theta_{j} \cos 2 j \tau$ is periodic with period $\pi$. The source term $\sigma(\tau)$ on the right-hand side of Eq. (C2) is zero for free perturbations (i.e. corresponding to adding some "eccentricity" to Hill's "circular-like" variational orbit), and non-zero when one perturbs

\footnotetext{
15 In Hénon's fourth paper [49], he gives the value $\pi m_{c r}=T / 2=0.61294$. The more precise value (C1) was privately communicated to us by Hénon, and follows also independently from our results in Appendix B (study of the geometric-like growth of various $m$-series and of the zeroes of Padé denominators).
} 
Hill's Hamiltonian $H_{\text {Hill }}=$ (kinetic terms) $+F_{0}+F_{2}$ (e.g. by adding the $F_{1}$ perturbation we are mainly concerned with, or the parallactic terms $\left.F_{3}+\cdots\right)$. Perigee precession is described by the general homogeneous solution of Eq. (C2) $(\sigma=0)$. The latter general homogeneous solution can be written as a linear combination of complex solutions of the form $q(\tau)=\zeta^{\mathrm{c}} \sum_{j} b_{j} \zeta^{2 j}$ and of their complex conjugates (we recall that $\zeta=e^{i \tau}$ ). On the one hand, the quantity c is linked to the usual rate of perigee advance $d \varpi / d t$ (in the non-rotating frame) by [17

$$
\mathrm{c}=\frac{d l}{d \tau}=(1+m)\left(1-\frac{1}{n} \frac{d \varpi}{d t}\right)
$$

(where $l=n t+\epsilon-\varpi$ is the "mean anomaly"). On the other hand, the quantity $\mathrm{c}$ is directly linked to the characteristic multipliers. Indeed, when $\tau \rightarrow \tau+2 \pi, q(\tau)$ gets multiplied by $e^{2 \pi i c}$, while $\bar{q}(\tau)$ gets multiplied by $e^{-2 \pi i c}$. Therefore the half-sum of the multipliers is simply

$$
a=\cos (2 \pi \mathrm{c}) .
$$

By using the perturbative series giving the perigee precession, $n^{-1}(d \varpi / d t)=\frac{3}{4} m^{2}+$ $\frac{177}{32} m^{3}+\cdots$ (which has been computed to high accuracy in Ref. [55]; see also [18]), one can check that the crossing of $a=+1$ found by Hénon corresponds, when $m$ increases from 0 to $m_{c r}$, to $\mathrm{c}$ increasing from 1 to a slightly higher value $(\simeq 1.1)$ and then decreasing to reach the value 1 at $m=m_{c r}$. From the smoothness of the variation of the characteristic multipliers, and therefore of $a$, with $m$ we deduce that, beyond $m=m_{c r}, \mathrm{c}(m)$ goes through a quadratic branch point $\mathrm{c}(m) \simeq\left(1-m / m_{c r}\right)^{1 / 2}$ and becomes complex .

Finally, the important information for our purpose is that when $m$ increases up to $m_{c r}$, the quantity $\mathrm{c}(m) \simeq\left(1-m / m_{c r}\right)^{1 / 2}$ is such that both functions $\cos 2 \pi \mathrm{c}(m)(=a)$ and $\cos \pi \mathrm{c}(\mathrm{m})$ cross smoothly (without branch points or discontinuities of derivatives) their corresponding limiting values $\cos 2 \pi=+1$ and $\cos \pi=-1$.

Let us now consider Hill's way of solving equation (C2). When inserting $q(\tau)=$ $\zeta^{\mathrm{c}} \sum_{j} b_{j} \zeta^{2 j}$ into (C2) one gets an infinite system of linear equations for the coefficients $b_{j}$. When written in a suitably normalized way, the determinant of this infinite system (which depends on c), say $\Delta(\mathrm{c})$, is a well-defined quantity (Hill's determinant). An homogeneous solution $(\sigma=0)$ exists only for the values of $\mathrm{c}$ for which $\Delta(\mathrm{c})=0$. On the other hand, if we consider the case where there is a source term on the right-hand side of Eq. (C2) of the form $\sigma(\tau)=\zeta^{\mathrm{c}_{\sigma}} \sum_{j} \sigma_{j} \zeta^{2 j}$, the corresponding inhomogeneous solution $q_{\text {inhom }}(\tau)$ will have the same form as $\sigma(\tau)$, but will contain, as for usual finite determinants, a factor $1 / \Delta\left(\mathrm{c}_{\sigma}\right)$. This factor will become infinite when $\mathrm{c}_{\sigma}$ tends to one of the free perigee precession values for which $\Delta(\mathrm{c})=0$. The analysis of the determinant $\Delta(\mathrm{c})=0$ [40], [17] shows that, as a function of $\mathrm{c}$,

\footnotetext{
${ }^{16}$ The combined facts that $m_{c r}$ is rather small and that $\mathrm{c}(m)$ has a quadratic branch point at $m=m_{c r}$ "explains" the notoriously bad convergence of the perturbation series giving $d \varpi / d t$. Rewriting this series in terms of better behaved quantities, such as $\cos (2 \pi c)$ or $\cos (\pi \mathrm{c})$, improves more its convergence than the "Euler transformations", $m \rightarrow m /(1+\alpha m)$, which have been traditionally used [56.
} 
it is a linear function of $\cos \pi \mathrm{c}$. We conclude that the forced perturbation $q_{\text {inhom }}(\tau)$ will contain the factor $\left(\cos \pi \mathrm{c}_{\sigma}-\cos \pi \mathrm{c}(m)\right)^{-1}$, where $\mathrm{c}(m)$ denotes the free perigee precession value. From our analysis above, we know that when $m$ crosses $m_{c r}$, $\cos \pi$ c crosses smoothly -1 . The final conclusion is that the source terms for which $\cos \pi \mathrm{c}_{\sigma}=-1$, i.e. $\mathrm{c}_{\sigma}= \pm 1, \pm 3, \pm 5, \ldots$, generates normal displacements of Hill's orbit which have pole singularities $\propto\left(m-m_{c r}\right)^{-1}$ as $m$ crosses the value (C1) found in linear stability analyses. Moreover, it is easily checked that the addition of a perturbing potential, say $F_{p}$, to Hill's potential $F_{0}+F_{2}$, generates a source term $\sigma(\tau)$ in Hill's variational equation (C2) which is a linear combination of $F_{p}$ and $\Re e\left[D u\left(\partial F_{p} / \partial u\right)\right]$ with real coefficients of the form $k_{0}+2 \sum_{j} k_{j} \cos 2 j \tau$. More precisely

$$
\sigma(\tau)=-2 \varphi\left\{\varphi^{2} \Phi F_{p}+\Re e\left[D u\left(\partial F_{p} / \partial u\right)\right]\right\}
$$

where

$$
\begin{aligned}
\varphi^{-2}(u, \bar{u}) & =-D u D \bar{u} \\
\Phi(u, \bar{u}) & =m D u D \bar{u}-2 \Re e\left[D u\left(\partial F_{\text {Hill }} / \partial u\right)\right],
\end{aligned}
$$

with $F_{\text {Hill }} \equiv F_{0}+F_{2}$.

Therefore, if $F_{p}=\Re e\left[\zeta^{\mathrm{c}_{p}} \sum_{j} f_{j} \zeta^{2 j}\right], \sigma$ will have the form $\sigma(\tau)=\Re e\left[\zeta^{\mathrm{c}_{p}} \sum_{j} \sigma_{j} \zeta^{2 j}\right]$. In other words, $\mathrm{c}_{\sigma}= \pm \mathrm{c}_{p}$, so that the perturbing potentials $F_{1}, F_{3}, F_{5}, \ldots$ generate source terms with $\mathrm{c}_{\sigma}= \pm 1, \mathrm{c}_{\sigma}= \pm 3, \mathrm{c}_{\sigma}= \pm 5, \ldots$, respectively.

\section{APPENDIX D: LAPLACE ON THE EQUIVALENCE PRINCIPLE}

In the first volume of his Traité de Mécanique Céleste [58 (presented to the French Academy of Sciences in 1799), Laplace lists a series of facts suggesting that gravity is proportional to the masses. This list (which is probably inspired by a corresponding list in the Principia, although Laplace does not mention Newton here) contains Newton's argument that the motion of satellites would be very sensitive to a violation of the universality of free fall, but does not quantify it. As far as we are aware, the only quantitative work of Laplace on this idea [5] is contained in the last book of the last volume of the Traité de Mécanique Céleste which was presented to the Académie des Sciences on 16 August 1825 [59].

The fact that Laplace was then 76 year old (he died a year and a half later on 5 March 1827) may explain why this work of Laplace contains some strange leaps of reasoning $\square$. It seems plausible to us that Laplace, when writing this chapter, was using previous notes of his which contained more detailed calculations and more consistent reasonings. Anyway, the aim of this Appendix is to show that the final limit he quotes on a possible violation of the equivalence principle,

$$
\left|\bar{\delta}_{12}\right|<\frac{1}{3410000} \simeq 2.9 \times 10^{-7}
$$

\footnotetext{
17 Basically he mentions that his lunar theory and combined (selected) lunar and solar parallax data agree to about $1.2 \%$ and then goes on to admit $1 / 8=12.5 \%$ as fractional upper limit on the theory/observation agreement.
} 
is a very reasonable (and slightly pessimistic) bound, which can be derived in a logically clear way using only the information Laplace had in hand.

Laplace's new idea (compared to Newton) was to use the "parallactic inequality" in the longitude of the Moon as a sensitive test of the equivalence principle" "Parallactic inequality" means the coefficient of the synodic term $-\sin \tau$ in the expression of the lunar longitude $v$ as a function of time TI. We have computed this coefficient, say $A$, in Appendices $\mathrm{A}$ and $\mathrm{B}$ for the Main Problem (i.e. neglecting eccentricities and inclinations). Its theoretical expression reads

$$
A^{\text {th }}=A_{\mathrm{PAR}}+A_{\mathrm{EP}}
$$

where the normal "parallactic" contribution reads

$$
A_{\mathrm{PAR}}=\frac{15}{8}\left(X_{2}-X_{1}\right) m \frac{\tilde{a}}{a^{\prime}} S_{\mathrm{PAR}}(m)
$$

while the "equivalence principle" one reads

$$
A_{\mathrm{EP}}=3 m \bar{\delta}_{12} \frac{a^{\prime}}{\tilde{a}} S_{\mathrm{EP}}(m)
$$

Here, $S_{\mathrm{PAR}}(m)=1+\frac{26}{5} m+\cdots, S_{\mathrm{EP}}(m)=1+\frac{16}{3} m+\cdots$, are slowly converging series in powers of $m=n^{\prime} /\left(n-n^{\prime}\right)$. In the third volume of his Traité de Mécanique Céleste, Laplace computes $A_{\text {PAR }}$ with particular care, pushing the calculation to fifth order in $m$ inclusively ${ }^{20}$. He was therefore entitled to considering that the theoretical error on $A_{\mathrm{PAR}}$ was negligible compared to the observational uncertainties in $A^{\text {obs }}$. Note that $A_{\mathrm{PAR}}$ is proportional to the inverse distance to the Sun, i.e. to the "solar parallax" $\pi_{S} \equiv R_{E} / a^{\prime}$, where $R_{E}$ is the equatorial radius of the Earth (hence the name "parallactic inequality"). The result of Laplace can be expressed as

$$
A_{\mathrm{PAR}} \simeq 14.3 \pi_{S}
$$

where both $A_{\mathrm{PAR}}$ and $\pi_{S}$ are expressed in seconds of $\operatorname{arc}^{27}$.

On the observational side, Laplace had in hand both lunar data and data on the solar parallax. The two phenomenological "lunar tables" he was using (one by Mason and one by

18 Note that as early as 1753 , T. Mayer had used the theory of the parallactic inequality to infer the value of the solar parallax [61].

19 Beware that Laplace was actually working with the inverse function: $t=t(v)$.

20 We have checked the first orders of his result and found them to agree with ours. Note that Laplace includes the effect of eccentricities and inclinations that we neglect.

${ }^{21}$ Beware that Laplace, in his volume III, uses (Révolution oblige) "decimal seconds", i.e. $10^{-6}$ of a right angle. 
Burg) gave for the "observed" value of the synodic inequality in longitude, $A_{\text {Mason }}^{\text {obs }}=116 . " 68$ and $A_{\text {Burg }}^{\text {obs }}=122 . " 38$ [60]. As for the solar parallax, many scientific expeditions had taken advantage of the passages of Venus in front of the Sun in 1761 and, especially, 1769 to measure $\pi_{S}$ (see 62] for a detailed text by a contemporary of Laplace, or [63] for a more exhaustive historical treatment). The published results ranged between $\pi_{S}^{\text {obs }}=8 . " 43$ (Planman) and $\pi_{S}^{\text {obs }}=8 . " 80$ (Pingré) [61, [63]. The comparison between the theoretical results (D2), (D5) and the observational results on $A$ and $\pi_{S}$ gives a value for a possible equivalence-principleviolation contribution

$$
A_{\mathrm{EP}}=A^{\mathrm{obs}}-14.3 \pi_{S}^{\mathrm{obs}}
$$

Worst-case limits on $A_{\mathrm{EP}}$ are obtained by taking the extreme values on the right-hand side of (D6) (e.g. $\left.A_{\mathrm{EP}}^{\max }=A_{\mathrm{Burg}}^{\mathrm{obs}}-14.3 \pi_{S}^{\mathrm{Planman}}\right)$ 22. This yields

$$
-9 . " 2<A_{\mathrm{EP}}<1 . " 8 \text {. }
$$

On the other hand, the theoretical result (D4) [using $a^{\prime} / \tilde{a} \simeq 391$ and $S_{\mathrm{EP}}(m) \simeq 1.72$ (see Appendix B)] reads $A_{\mathrm{EP}} \simeq 163 \bar{\delta}_{12} \simeq 3.36 \bar{\delta}_{12} \times 10^{7}$ in seconds of arc, so that we get the following worst-case bounds on $\bar{\delta}_{12}$

$$
-2.7 \times 10^{-7}<\bar{\delta}_{12}<0.54 \times 10^{-7}
$$

From this point of view, the final bound quoted by Laplace, Eq. (D1), seems very reasonable and consistent with the observational uncertainties in his time. Note, however, that Laplace never quotes a precise theoretical formula for $A_{\mathrm{EP}}$. He only says (and uses) the fact that the synodic amplitude $A$ is proportional to its source term in the perturbing function. This neglects the leading " $p$ dependence" of $C_{v} \propto 2+p$ in Eq. (A19b) which says that $A_{\mathrm{EP}} / A_{\mathrm{PAR}} \propto$ $3 / 5 \times S_{\mathrm{EP}}(m) / S_{\mathrm{PAR}}(m)$. In Laplace's published analysis the lacking (unfavourable) factor $3 / 5$ is effectively compensated by his overpessimistic estimate of the fractional uncertainty on $A: 1 / 8=12.5 \%$.

Let us end this section by raising a historical question. Though Laplace was fully aware of the scientific interest of the bound (D1), and of the fact that it was (at the time) more precise than the bounds obtained from ground tests of the universality of free fall, his successors in celestial mechanics seem (as far as we know) to have lost interest in the issue. However, near the end of the nineteenth century, especially after the theoretical work of Hansen, Delaunay, Hill and Brown (who improved the computation of $A_{\mathrm{PAR}}^{\text {th }}$ ), and after many improvements on the observational side, it should have been possible to obtain more stringent limits on $\bar{\delta}_{12}$. For instance, Delaunay computed $A_{\mathrm{PAR}}^{\text {th }}$ to $m^{7}$ which corresponds to a truncature error

\footnotetext{
22 Such a worst-case approach seems appropriate to a pre-least-squares-law period. Before Gauss' theory of measurement errors, scientists quoted only "central values" for measured quantities.

23 As for the subleading dependence on $p$, i.e. the ratio $S_{\mathrm{EP}}(m) / S_{\mathrm{PAR}}(m) \simeq 1.72 / 1.60 \simeq 1.08$ Laplace's experience with similar factors in many terms of lunar theory might have suggested him that he did not need to worry about it.
} 
of $0 . " 3366 / 125 . " 4201 \simeq 2.7 \times 10^{-3}$ [55] (for the principal part of $A$; see also Appendix B). This is negligible (when added in quadrature) compared to the observational error on $\pi_{S}$ at the time. For instance, the laboratory measurements of the velocity of light by Foucault and Cornu gave values ranging between $\pi_{S}=8 . " 834$ and $\pi_{S}=8 . " 881$, the passage of Venus across the solar disk in 1874 gave a range 8." $76-8$. " 88 [61], and the recommended value starting in 1896 was 8." 80 . This suggests that a reasonable upper bound on the uncertainty of $\pi_{S}$ at that time was $0 . " 08$ (i.e. 0.9\%). [By comparison, the modern value is $\pi_{S}=8 . " 794148$ [48.] On the other hand, the observational error on $A^{\text {obs }}$ at the time was $<\frac{1}{2}(125 . " 46-124 . " 70)=0$." 38, i.e. $<0.3 \%$ (see [61], p. 533). This has a negligible effect on the derivation of a bound on $\bar{\delta}_{12}$. Using Eq. (D6) with $A_{\mathrm{EP}} \simeq 3.36 \bar{\delta}_{12} \times 10^{7}$ seconds of arc, this leads to the bound $\left|\bar{\delta}_{12}\right|<14.3 \delta \pi_{S}^{\text {obs }} /\left(3.36 \times 10^{7}\right) \simeq 3.4 \times 10^{-8}$, which is slightly better than the value obtained by Eötvös in $1890\left(\left|\bar{\delta}_{A B}\right|<5 \times 10^{-8}[7]\right)$. We do not wish to take too seriously such a posteriori derivations of limits on $\bar{\delta}_{12}$, but we consider this as an interesting example of historical eclipse of a deep concept, which has been rejuvenated, within a new theoretical and observational context, only in the last decades

\footnotetext{
${ }^{24}$ Let us note for completeness that the determinations of the mass of the Moon at the time were accurate enough to estimate with negligible error the mass ratio factor $X_{2}-X_{1}$ entering $A_{\mathrm{PAR}}$.

${ }^{25}$ For completeness, let us note that Poincaré kept alive this concept by mentioning Laplace's result in one of his popular books 64].
} 


\section{REFERENCES}

* On leave from the Institute of Astronomy, Charles University, Švédská 8, 15000 Prague 5, Czech Republic (e-mail: davok@aci.cvut.cz).

[1] G. Galilei, Discorsi e dimostrazione matematiche intorno à due nuove scienze, (Elzevir, Leiden, 1638); French translation by M. Clavelin, Discours concernant deux sciences nouvelles (A. Colin, Paris, 1970); see p. 61: “... it came to my mind that, if the resistance from the medium were totally suppressed, all bodies would go down with the same speed."; see also p. 70 where Galileo claims to have verified the universality of free fall, to better than $0.1 \%$, by pendulum experiments. The pages quoted here correspond, respectively, to p.116 and p.129 of the Edizione nazionale, and to p.72 and p.85 of the English translation by H. Crew and A. de Salvio, Dialogues concerning two new sciences, (Dover, New York, 1954).

[2] Though this issue has a long prehistory (starting, say, with J. Philoponos, 5th Century A.D.), even G.B. Benedetti who came closest to stating clearly the universality of free fall (in his Resolutio of 1553, and his Demonstratio of 1554) did not quite succeed in doing so. See Mechanics in Sixteenth-Century Italy; Selections from Tartaglia, Benedetti, Guido Ubaldo and Galileo, translated and annotated by S. Drake and I.E. Drabkin (The University of Wisconsin Press, Madison, 1969).

[3] I. Newton, Philosophiae Naturalis Principia Mathematica (London, 1687); English translation by F. Cajori, Newton's Principia (University of California Press, Berkeley, 1934).

[4] See Book III, Proposition VI, Theorem VI of the Principia Mathematica, pp. 411413 of Cajori's edition; see also the discussion in T. Damour, chapter 6 of 300 years of gravitation, edited by S.W. Hawking and W. Israel (Cambridge University Press, Cambridge, 1987), pp. 128-198, notably the Section 6.6, pp. 141-144.

[5] P.S. Laplace, Traité de Mécanique Céleste, Volume V (Bachelier, Paris, 1825). Laplace's work is contained in Book XVI, Chapter IV. Reprinted in Oeuvres de Laplace, Tome Cinquième (Gauthier-Villars, Paris, 1882), pp. 445-452.

[6] F.W. Bessel, Ann. der Physik und Chemie (Poggendorff) 25, 401 (1832).

[7] R. v. Eötvös, Math. u. Naturw. Ber. aus Ungarn 8, 65 (1890).

[8] R. v. Eötvös, D. Pekár and E. Fekete, Ann. der Physik 68, 11 (1922).

[9] A. Einstein, Jahrb. Radioact. Elekt. 4, 411 (1907).

[10] K. Nordtvedt, Phys. Rev. 170, 1186 (1968).

[11] K. Nordtvedt, Phys. Rev. 169, 1014 (1968).

[12] K. Nordtvedt, Phys. Rev. 169, 1017 (1968).

[13] R.H. Dicke, Rev. Mod. Phys. 29, 355 (1957); see notably pp. 356, 357 in which by "weak interaction energy" Dicke means both Fermi interactions and gravitation.

[14] R.H. Dicke, in Evidence for Gravitational Theories, Proceedings of the International School of Physics "Enrico Fermi", Course XX, edited by C. Møller (Academic Press, New York, 1962), pp. 1-49, see notably pp. 16-19 and 26-27.

[15] R.H. Dicke, in Gravitation and Relativity, edited by H.Y. Chiu and W.F. Hoffman (Benjamin, New York, 1964), pp. 1-16, see notably pp. 13-16.

[16] K. Nordtvedt, Phys. Rev. D 7, 2347 (1973). 
[17] D. Brouwer and G.M. Clemence, Methods of Celestial Mechanics, (Academic Press, Orlando, 1961).

[18] V.A. Brumberg and T.V. Ivanova, Trans. Inst. Theor. Astron. (Leningrad) 19, 3 (1985).

[19] C.M. Will, Theory and Experiment in Gravitational Physics, (Cambridge University Press, Cambridge, 1981); second edition 1993.

[20] J.G. Williams et al., Phys. Rev. Lett. 36, 551 (1976).

[21] I.I. Shapiro et al., Phys. Rev. Lett. 36, 555 (1976).

[22] J.O. Dickey, X.X. Newhall and J.G. Williams, Adv. Space Res. 9, No. 9, 75 (1989).

[23] J. Müller, M. Schneider, M. Soffel and H. Ruder, Astroph. J. 382, L101 (1991).

[24] G. de Pontécoulant, Théorie analytique du système du monde, Volume IV, (Paris, 1846)

[25] K. Nordtvedt, Icarus 114, 51 (1995).

[26] W. de Sitter, Mon. Not. R. Astr. Soc. 76, 699; 77, 155 (1916).

[27] V.A. Brumberg, Bull. Inst. Theor. Astron. (Leningrad, USSR) 6, 733 (1958).

[28] M.H. Soffel, Relativity in Astrometry, Celestial Mechanics and Geodesy, (Springer, Berlin, 1989).

[29] V.A. Brumberg, Essential Relativistic Celestial Mechanics, (Adam Hilger, Bristol, 1991).

[30] J.F. Lestrade and M. Chapront-Touzé, Astron. Astroph. 116, 75 (1982).

[31] M. Soffel, H. Ruder and M. Schneider, Astron. Astroph. 157, 357 (1986).

[32] B. Shahid-Saless, Phys. Rev. D 46, 5404 (1992).

[33] J.G. Williams, X.X. Newhall and J.O. Dickey, Phys. Rev. D, in press.

[34] T. Damour and G. Esposito-Farèse, Phys. Rev. D 50, 2381 (1994).

[35] T. Damour, in Gravitation and Quantizations, Proceedings of the LVIIth Les Houches Summer School, edited by B. Julia and J. Zinn-Justin (North-Holland, Amsterdam, 1995), pp. 1-62 .

[36] T. Damour and A.M. Polyakov, Nucl. Phys. B 423, 532 (1994); and Gen. Rel. Grav. 26, 1171 (1994).

[37] T. Damour and G. Esposito-Farèse, Class. Quantum Grav. 9, 2093 (1992).

[38] T. Damour, M. Soffel and C. Xu, Phys. Rev. D 43, 3273 (1991); 45, 1017 (1992); 47, 3124 (1993); 49, 618 (1994); see also T. Damour and D. Vokrouhlický, submitted to Phys. Rev. D.

[39] G.W. Hill, Amer. J. Math. 1, 5 (1878); 1, 129 (1878); 1, 245 (1878); Acta Math. 8, 1 (1886).

[40] E.W. Brown, An Introductory Treatise on the Lunar Theory, (Cambridge University Press, Cambridge, 1896); reprinted by (Dover, New York, 1960).

[41] A. Wintner, Math. Z. 24, 259 (1926).

[42] Y. Hagihara, Celestial Mechanics, Volume II, Part 2 (The MIT Press, Cambridge, 1972).

[43] C.L. Siegel and J.K. Moser, Lectures on Celestial Mechanics, (Springer-Verlag, Berlin, 1971).

[44] A.M. Liapunov, Trudi Otd. Fys. Nauk Ljobit. Estestvoznan. 8, 1 (1896); reprinted in Liapunov Sobranie Sochinenii I, 418 (Akad. Nauk SSSR, 1954).

[45] H. Poincaré, Leçons de Mécanique Céleste, Volume 2, Part 2, (Gauthier-Villars, Paris, 1909).

[46] M. Moons, MINIMS: User's guide, (Rapport interne No. 91/15 du Département de Mathématique FUNDP, Namur, 1991). 
[47] M.C. Gutzwiller and D.S. Schmidt, Astronomical Papers prepared for the use of the American Ephemeris and Nautical Almanach, Volume XXIII, Part I (1986).

[48] Connaissance des Temps pour l'An 1995, Bureau des Longitudes, (Service Hydrographique et Océanographique de la Marine, Brest , 1994).

[49] M. Hénon, Ann. Astr. 28, 499 (1965); 28, 992 (1965); Bull. Astr. Paris 1, fasc. 1, 57 (1966); 1, fasc. 2, 49 (1966); Astron. Astroph. 1, 223 (1969); Astron. Astroph. 9, 24 (1970). Hill's problem is treated in the last two references.

[50] J.O. Dickey et al., Science 265, 482 (1994).

[51] J. Laskar and O. Néron de Surgy, private communication (1995).

[52] Y. Su et al., Phys. Rev D 50, 3614 (1994).

[53] T. Damour and K. Nordtvedt, Phys. Rev. Lett. 70, 2217 (1993); Phys. Rev. D 48, 3436 (1993).

[54] B. Chauvineau and F. Mignard, Atlas of the Circular Planar Hill's problem, (Internal Publication of the Observatoire de la Côte d'Azur, Grasse, 1991).

[55] A. Deprit, J. Henrard and A. Rom, Astron. J. 76, 273 (1971).

[56] J. Henrard, Celest. Mechanics 17, 195 (1978); Ciel et Terre, 1-27 (1973).

[57] V.I. Arnold, Mathematical Methods of Classical Mechanics, (Springer-Verlag, New York, 1989).

[58] P.S. Laplace, Traité de Mécanique Céleste, Volume I (Paris, 1799), first part, Book II. Reprinted in Oeuvres de Laplace, Tome Premier (Imprimerie Royale, Paris, 1843), p. 139.

[59] C.C. Gillipsie, editor, Dictionary of Scientific Bibliography, Volume XV, Supplement I (Scribner, New York, 1970), pp. 387-403.

[60] P.S. Laplace, Traité de Mécanique Céleste, Volume III (Paris, 1802), Book VII. Reprinted in Oeuvres de Laplace, Tome Troisième (Imprimerie Royale, Paris, 1844), pp. 196-327.

[61] J.C. Houzeau, Vace-Mecum de l'Astronome, Annales de l'Observatoire Royal de Bruxelles, (F. Hayez, Bruxelles, 1882), pp. 403-411.

[62] J. Lalande, Astronomie, Tome Second, (Didot l'Aîné, Paris, 1792).

[63] H. Woolf, The transits of Venus, (Princeton University Press, Princeton, 1959).

[64] H. Poincaré, Science et Méthode (Flammarion, Paris, 1908); see book III, chapter III, section III. 


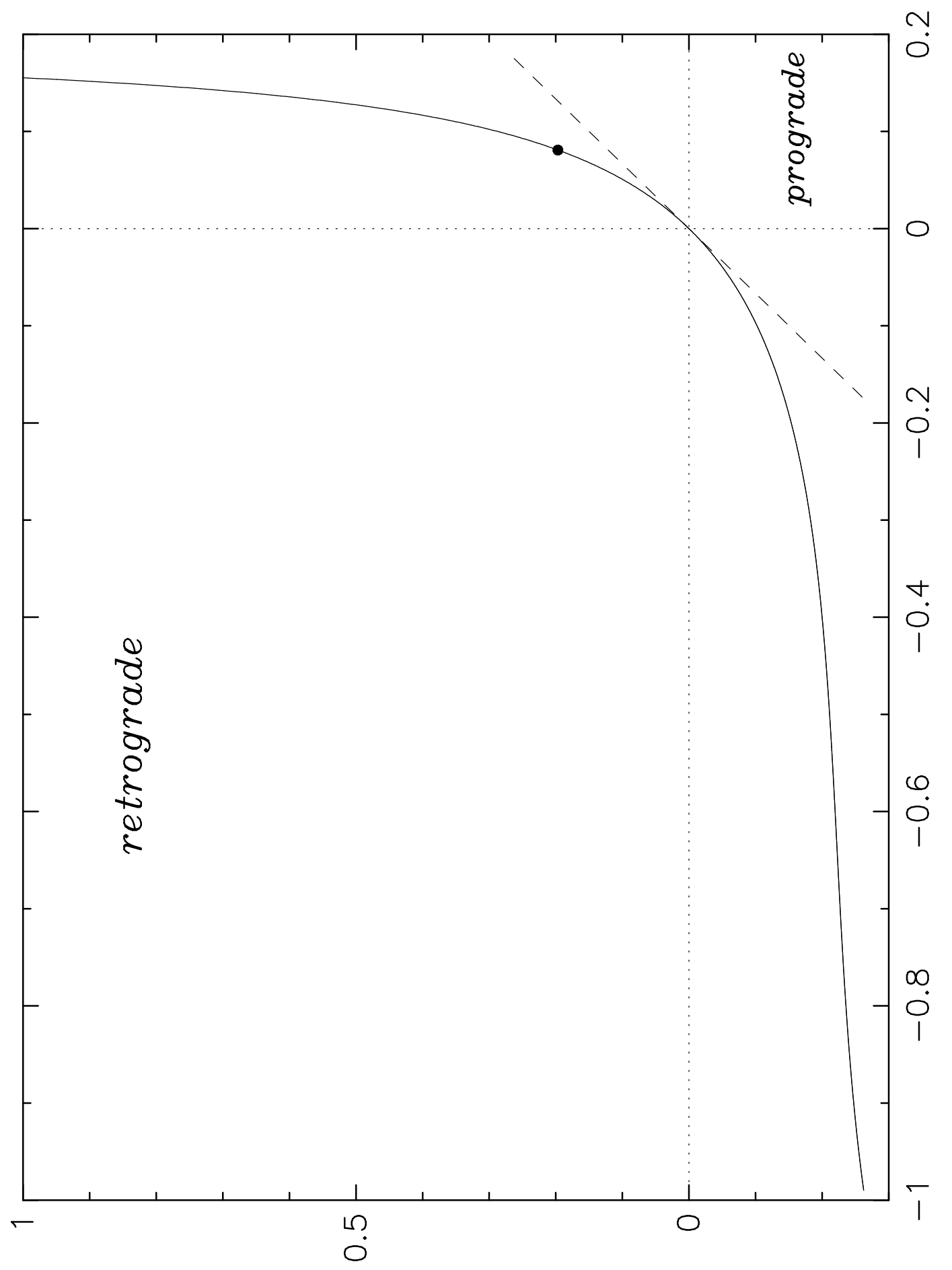

